\title{
New radiative transfer models for obscuring tori in active galaxies
}

\author{
I. M. van Bemmel ${ }^{1}$ and C. P. Dullemond ${ }^{2}$
}

\author{
1 Kapteyn Astronomical Institute, PO Box 800, 9700 AV Groningen, The Netherlands \\ 2 Max-Planck Institut für Astronomie, PO Box 1317, 85741 Garching, Germany
}

Received 10 June 2002 / Accepted 17 March 2003

\begin{abstract}
Two-dimensional radiative transfer is employed to obtain the broad-band infrared spectrum of active galaxies. In the models we vary the geometry and size of the obscuring medium, the surface density, the opacity and the grain size distribution. Resulting spectral energy distributions are constructed for different orientations of the toroid. Colour-colour comparisons with observational data are consistent with previous observations that the emission longward of $60 \mu \mathrm{m}$ is produced by star-formation and unrelated to the presence of an obscuring torus. We also find that the toroid cannot be flat, but is rather conical or flaring. The density is most likely constant with radius, and the size is relatively large with an inner radius around $10 \mathrm{pc}$. A direct comparison with radio galaxy Cygnus A yields a best fit for a conical disk with constant surface density, and a size from 10 to $30 \mathrm{pc}$, assuming the far-infrared emission is due to star-formation in the host galaxy.
\end{abstract}

Key words. galaxies: active - galaxies individual: Cygnus A - infrared: galaxies - infrared: ISM - quasars: general ISM: dust

\section{Introduction}

Unified models for active galaxies postulate the existence of a circumnuclear dust torus. This torus obscures the central regions from view at certain solid angles, at wavelengths where the dust is optically thick. Various authors have studied the behaviour of a dusty torus in the presence of a strong radiation field. Models have been constructed for the absorption of the optical to FUV emission by the dust, and the re-emission of this energy in the infrared (Pier \& Krolik 1992; Granato \& Danese 1994; Efstathiou \& Rowan-Robinson 1995). These models have been used to interpret IRAS observations of active galaxies. Their main result is that the spectrum from a dusty disk is much broader than a single grey body. However, observed SEDs are often still broader than the models, and show different behaviour of the $10 \mu \mathrm{m}$ silicate feature.

As discussed in previous work (van Bemmel \& Barthel 2001), it is very likely that there are two dust components in active galaxies. One component is a large scale dust distribution, heated by stars. The other is the obscuring torus. In narrowline radio galaxies and quasars it is difficult to disentangle the two components, since their broad-band spectra are not well known, due to their distance and the sensitivity of previous infrared instruments. Previous model studies of obscuring tori were only concerned with another subset of the active galaxies, namely Seyfert galaxies, which happen to be nearby and therefore bright and well observed infrared sources. Moreover, these studies focused on reproducing the general width of the SED.

Send offprint requests to: I. M. van Bemmel,

e-mail: bemmel@stsci.edu
Furthermore, the emphasis was on fitting a few selected objects, rather than a complete sample. In previous studies, the model geometry has been fixed, and the dependence of the resulting SED on the geometry and other disk parameters is not well understood. Finally, the models assume standard Galactic dust, which might not be appropriate for luminous active galaxies. Several studies (e.g. Laor \& Draine 1993; Maiolino et al. 2001a,b) have shown that the lack of a $10 \mu \mathrm{m}$ silicate emission feature and shallow $2200 \AA$ absorption can point to dust that is dominated by relatively large grains, while Galactic dust is dominated by small grains.

More recent models treat the torus as a clumpy medium, which produces a broader infrared spectrum, more consistent with the observations (Nenkova et al. 2002). However, these authors do not vary the geometry of the torus, nor the distribution of the mass. Although it is likely that the torus does not have a smooth density distribution, it is not yet understood how other parameters influence the width of the resulting broadband spectrum. Moreover, they assume that all infrared emission observed in active galaxies arises in the torus.

Here we will re-examine the infrared SEDs of active galaxies from a theoretical point of view. We employ a new and very versatile radiative transfer code RADICAL (Dullemond \& Turolla 2000), and examine a wide parameter space. Specifically, we study the dependence of the emission on observing angle, optical depth, disk geometry and size, dust mass distribution and dust grain size distribution. The initial goal will be to constrain the parameters of the torus, by comparing the colours to observations, and by fitting the infrared broad-band spectrum of Cygnus A. As a first order approximation we will 
Table 1. The different values for the model parameters, and their value in the reference model. The inclination $\theta$ is defined with respect to the symmetry axis of the torus.

\begin{tabular}{lccc}
\hline \hline Parameter & Symbol & Values & $\begin{array}{c}\text { Reference } \\
\text { model }\end{array}$ \\
\hline Equatorial optical depth at $20 \mu \mathrm{m}$ & $\tau_{20}$ & $5,10,20,50$ & 10 \\
Scale height exponent & $\alpha$ & $0,1,9 / 7$ & 1 \\
Surface density exponent & $\beta$ & $0,-1,-2$ & 0 \\
Inner radius of the disk (pc) & $r_{0}$ & 3,30 & 3,30 \\
Outer radius of the disk (pc) & $r_{\text {out }}$ & 30,300 & 30,300 \\
Aspect angle (degrees) & $\theta$ & $5,30,50,60,70,90$ & all \\
Grain size exponent & $q$ & $-2,-3.5$ & -2 \\
Scale height at inner radius (pc) & $h_{0}$ & $=r_{0}$ & 3 \\
Spectral index of input spectrum & $j$ & -2 & -2 \\
$100-400$ eV luminosity & $L_{X}$ & $10^{12} L_{\odot}$ & $10^{12} L_{\odot}$ \\
\hline
\end{tabular}

assume the torus has a smooth distribution of dust. Eventually, these new models can be used to disentangle the two dust components in active galaxies, which will allow us to study starformation in active galaxies, and the parameters of the obscuring torus in more detail than before.

\section{Model setup}

In our disk model a geometry for the dust, an input spectrum and dust opacities need to be specified. The numerical method is described below in Sect. 3. We have constructed a grid of models, built by varying different parameters. This results in a set of over 150 model SEDs, in which all variables are crosscorrelated. Below we describe the different parameters of the models (see also Table 1).

\subsection{Disk geometry and density distribution}

The density of the disk in angular direction is a Gaussian, with a width of $h(r)$. The mass density for any point in the disk is then given by:

$\rho(r, \theta)=\frac{\Sigma(r)}{h(r)} \sqrt{\pi} \cdot \exp \left[-\left(\frac{z}{h(r)}\right)^{2}\right]$

with $z=r * \tan (\theta)$ and $\Sigma(r)$ the surface density. Due to the setup of the model, the surface where $\tau_{20}$ equals unity does not coincide with the scale height of the disk. For higher opacities this surface lies at higher $z$. Therefore the actual opening angle of the torus is smaller for higher opacities, although the width of the Gaussian is kept constant.

The geometry of the disk is thus described by four parameters: the scale height $h(r)$, the surface density $\Sigma(r)$, the inner radius $r_{0}$ and the outer radius $r_{\text {out }}$. Each of these parameters is varied. For the scale height we assume:

$h(r)=h_{0}\left(\frac{r}{r_{0}}\right)^{\alpha}$ and the surface density is defined as:

$\Sigma(r)=\Sigma_{0}\left(\frac{r}{r_{0}}\right)^{\beta}$

with $r_{0}$ the inner radius of the disk.

For $h_{0}$ we assume it equals the inner radius, i.e. $h\left(r_{0}\right)=$ $h_{0}=r_{0}$, so the disk is thicker if it is further away from the emission source. This implies that the opening angles are comparable for similar values of $\tau_{20}$. The resulting opening angles are consistent with the size of the broad-line region that has to be shielded in a more edge-on orientation, and with the observed opening angles of ionization cones in active galaxies.

The exponents $\alpha$ and $\beta$ are variable model parameters. They determine the geometry and radial density distribution. We consider a pillbox disk $(\alpha=0)$, a conical disk $(\alpha=1)$ or a flared disk $(\alpha=9 / 7)$, see also Fig. 1 . The flaring exponent of $9 / 7$ is chosen following models for young stellar objects (Chiang \& Goldreich 1997), since there are no better constraints for disks around active galactic nuclei. $\beta$ determines the surface density distribution, i.e. the mass concentration with radius. We constructed models with a flat mass distribution $(\beta=0)$, which implies that the mass is concentrated in the outer regions for the conical and flaring disk. In addition, we have models where the mass is concentrated in the inner regions, either similar amounts in logarithmic intervals $(\beta=-2)$, or less centrally concentrated $(\beta=-1)$.

The radial optical depth of the disk at the equator is defined at $20 \mu \mathrm{m}\left(\tau_{20}\right)$, and four values are used: 5, 10, 20 and 50. A value below 1 at $20 \mu \mathrm{m}$ is inconsistent with observed anisotropies in the mid-infrared (van Bemmel et al. 1998, 2000; Heckman et al. 1992). The upper value is limited by the numerical possibilities of RADICAL. $\Sigma_{0}$ is chosen such that the model has the desired radial equatorial optical depth at $20 \mu \mathrm{m}$.

The inner radius $\left(r_{0}\right)$ is either 3 or $30 \mathrm{pc}$, the outer radius $\left(r_{\text {out }}\right) 30$ or $300 \mathrm{pc}$, which results in three different sizes for the disk. These will be referred to as large disk $(L=3-300 \mathrm{pc})$, inner disk $(I=3-30 \mathrm{pc})$ and outer disk $(O=30-300 \mathrm{pc})$. 

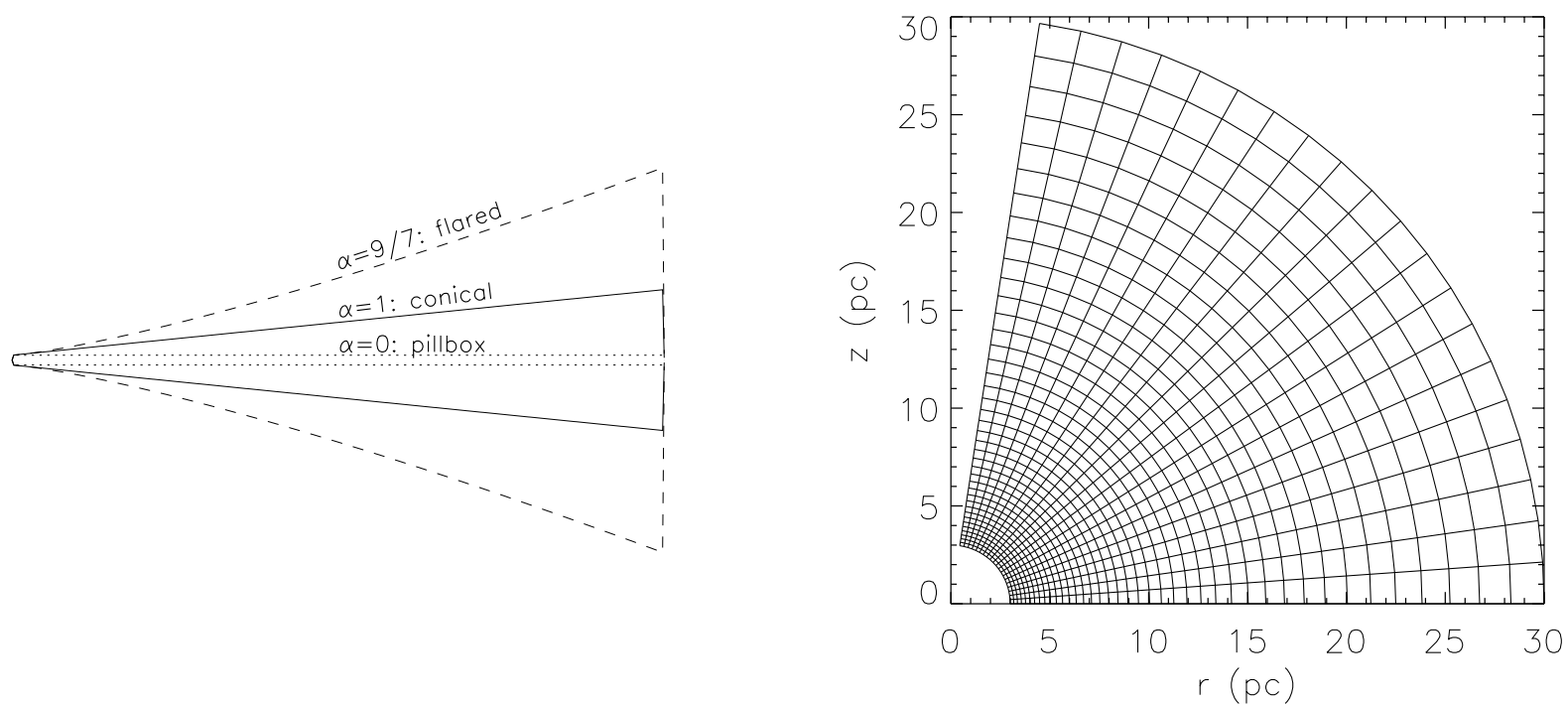

Fig. 1. Left panel: the three different geometries of the dust disk. Dotted line is the pillbox model $(\alpha=0)$, solid line the conical disk $(\alpha=1)$, and the dashed line is the flared disk $(\alpha=9 / 7)$. Right panel: an illustration of the grid setup in Cartesian coordinates. The grid is mirrored in the $r$-axis and rotated on the $z$-axis to achieve full spatial coverage of the dust disk. Refinement of the grid cells at small radii is not shown.

\subsection{Dust characteristics}

Using the extinction efficiency tables from Laor \& Draine (1993) we have constructed an input opacity table. The mass absorption coefficient $\kappa_{\lambda}$ is obtained by summing the values of $\kappa_{\lambda}(a)$ for all separate grain sizes and dust species, using a normalized size distribution $S_{i}(a)$ for each dust species. The normalized size distribution is given by:

$S_{i}(a)=\frac{a^{q} m_{\mathrm{d}, i} X_{i}}{\sum_{a} S_{i}(a)}$

where $a$ is the grain radius, $m_{\mathrm{d}, i}$ the mass of a dust grain of dust species $i, X_{i}$ the fraction of dust species $i$ and $q$ a dimensionless exponent that can vary between -0.5 and -3.5 . In Fig. 3 the effect of varying $q$ on the resulting opacity is shown. We use only graphite and silicate dust in a $50 / 50 \%$ ratio $\left(X_{i}=0.5\right)$. The grain sizes range from 0.001 to $10 \mu \mathrm{m}$.

For $q=-3.5$ the Standard Galactic Dust or MRN dust is obtained, as described by Mathis et al. (1977). We have made models with $q=-3.5$ and $q=-2.0$ to test the effect of the grain size distribution on a conical disk $(\alpha=1)$ with a constant density $(\beta=0)$. No silicate $10 \mu \mathrm{m}$ emission feature has yet been detected in any active galaxy, constituting a major problem for radiative transfer models of obscuring tori so far. The lack of $10 \mu \mathrm{m}$ emission might indicate that dust in active galaxies is dominated by larger grains than Galactic dust (Laor \& Draine 1993). Since other studies point in the same direction (Maiolino et al. 2001b), we have adopted $q=-2.0$ as standard.

\subsection{Input spectrum}

The input spectrum is defined from $10^{11}$ to $10^{17} \mathrm{~Hz}$ i.e. from $3 \mathrm{~mm}$ to $3 \mathrm{~nm}(400 \mathrm{eV})$, and divided in 112 equally large logarithmic spacings. This number was chosen to optimize both calculation speed and frequency resolution. The upper limit for the frequency lies at the point where the dust opacity drops precipitously and the exact value is of little consequence.
The lower limit lies far enough in the millimeter to ensure complete coverage of the infrared output spectrum.

For the shape of the input spectrum a power-law is assumed:

$L(v)=L_{0} v^{j}$

This allows for two more variables: the spectral slope $j$ and the total luminosity. The results do not depend strongly on $j$ when it is varied between 0 and 4 ; we will adopt $j=2$. The luminosity is scaled to be $10^{12} L_{\odot}$, integrated over the highest energies $(100-400 \mathrm{eV})$, which is a typical intrinsic X-ray luminosity for radio-loud active galaxies. In Fig. 2 we show the spectrum that we use as input for RADICAL.

\subsection{Grid setup}

The radial grid is set up using $r_{0}$ and $r_{\text {out }}$. A fixed number of radial points is defined between the inner and outer radius. The grid is refined at the inner edge to prevent flux overshoots in the innermost grid cells. For the small disks, we have used 40 radial gridpoints; for the large disk 60 . The angular grid does not need to extend from pole to pole. The dust density in the wings of the Gaussian is negligible at a few scale heights, and the disk can be mirrored in the equator. The grid is therefore defined from the equator to a maximum angle that extends well beyond the height where the opacity reaches unity. The code assumes that the remaining space has the same density as the grid cells on the edge of the defined angular raster. We use 20 gridpoints in angular direction for all models. The resulting grid is depicted in Fig. 1.

\section{The radiative transfer code RADICAL}

In order to compute the spectrum emitted by the irradiated torus, a $2 \mathrm{D}$ radiative transfer calculation is performed. 

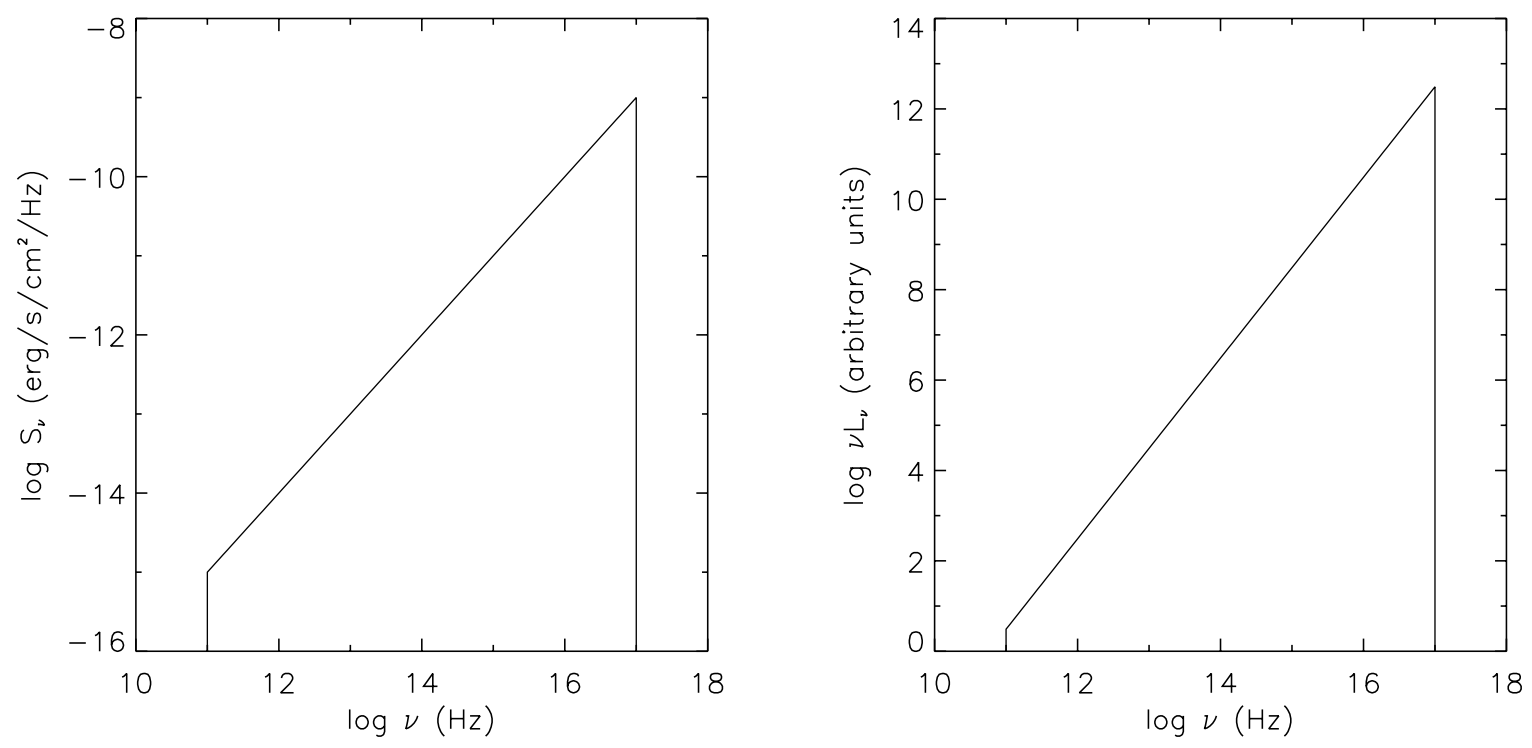

Fig. 2. The input spectrum used for the models. On the left the flux as it is used by RADICAL, on the right the matching energy spectrum, using $j=2$.

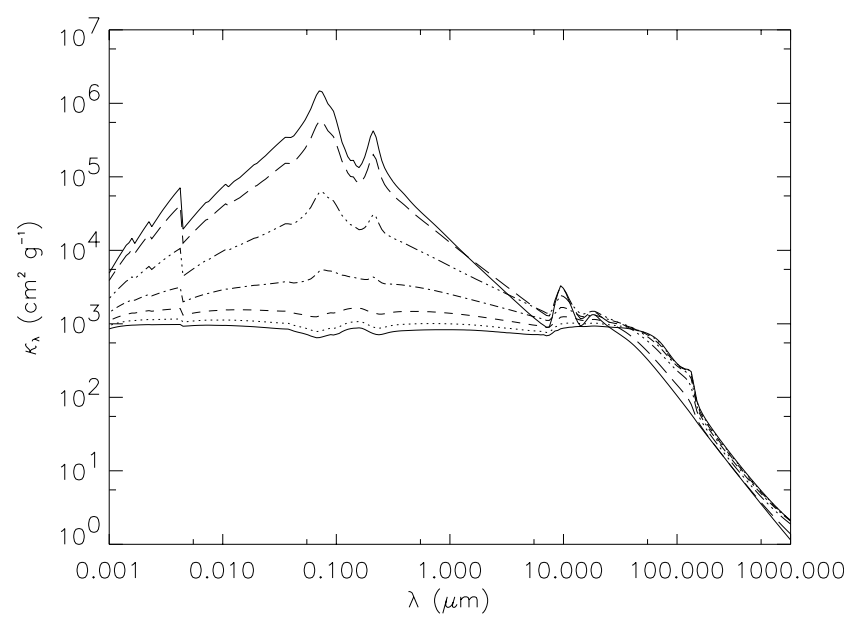

Fig. 3. The resulting opacities for different values of $q$, the exponent of the grain size distribution. From top to bottom $q$ increases from -3.5 to -0.5 with steps of 0.5 . For the models we used $q=-2$ (dashdotted line). The frequency resolution of this plot is higher than for the models.

This is done using RADICAL ${ }^{1}$, which is an all-purpose 2D radiative transfer code for axisymmetric circumstellar media (Dullemond \& Turolla 2000). In this section we describe how we use this code to solve the transfer problem of a torus, partially obscuring the active nucleus.

The dust in the torus is assumed to be in equilibrium with the radiation field. Each dust grain has a fine-tuned temperature such that it emits as much energy per second as it absorbs. In the case of an optically thin torus this temperature is easily computed, since the flux of the nucleus is known and simply goes as $1 / r^{2}$. In such a case, no radiative transfer is needed, since the temperatures of the grains can be computed.

\footnotetext{
${ }^{1}$ For more information, see also http://www.mpa-garching. mpg.de/PUBLICATIONS/DATA/radtrans/radical/
}

A simple volume integral over the emission is then sufficient to obtain the emitted spectrum.

Observations of radio-loud active galaxies suggest that the opacity of the obscuring disk exceeds unity at least up to midinfrared wavelengths $(\sim 40 \mu \mathrm{m})$. The calculation of the emission changes significantly in optically thick models. The nuclear radiation is now stopped when it is intercepted by the torus at the inner edge, and slowly diffuses into the dust. The inner edge is therefore expected to be very hot, while the interior of the torus is much cooler. The spectral energy distribution of such a torus with a large range in temperatures will be broader than expected from an optically thin torus. Solving the temperature of the dust grains is much more difficult than in the optically thin case. This temperature depends not only on the incident nuclear flux, but also on the infrared radiation of the torus itself. A grain located many optical depths deep into the torus will see little of the direct nuclear radiation, but instead will see the infrared emission of its neighboring grains. The radiative equilibrium of this grain depends therefore on the emission of the grains elsewhere in the torus. Every element of the torus is radiatively connected to every other part.

\subsection{The transfer algorithm}

At present, the best way to solve this problem is to perform a $2 \mathrm{D}$ radiative transfer calculation. The absorption and re-emission of radiative energy will be modeled as it seeps through the torus. The radiative transfer along each ray through the torus is given by:

$\frac{\mathrm{d} I_{v}}{\mathrm{~d} s}=\rho \kappa_{v}\left(B_{v}(T)-I_{v}\right)$

where $B_{v}(T)$ is the Planck function at the local temperature, $I_{v}$ the intensity, and $s$ the path length along the ray. The solution of the radiative transfer proceeds as follows. We start with an initial guess for the dust temperature distribution. Using a radiative transfer integrator based on the algorithm of Extended 

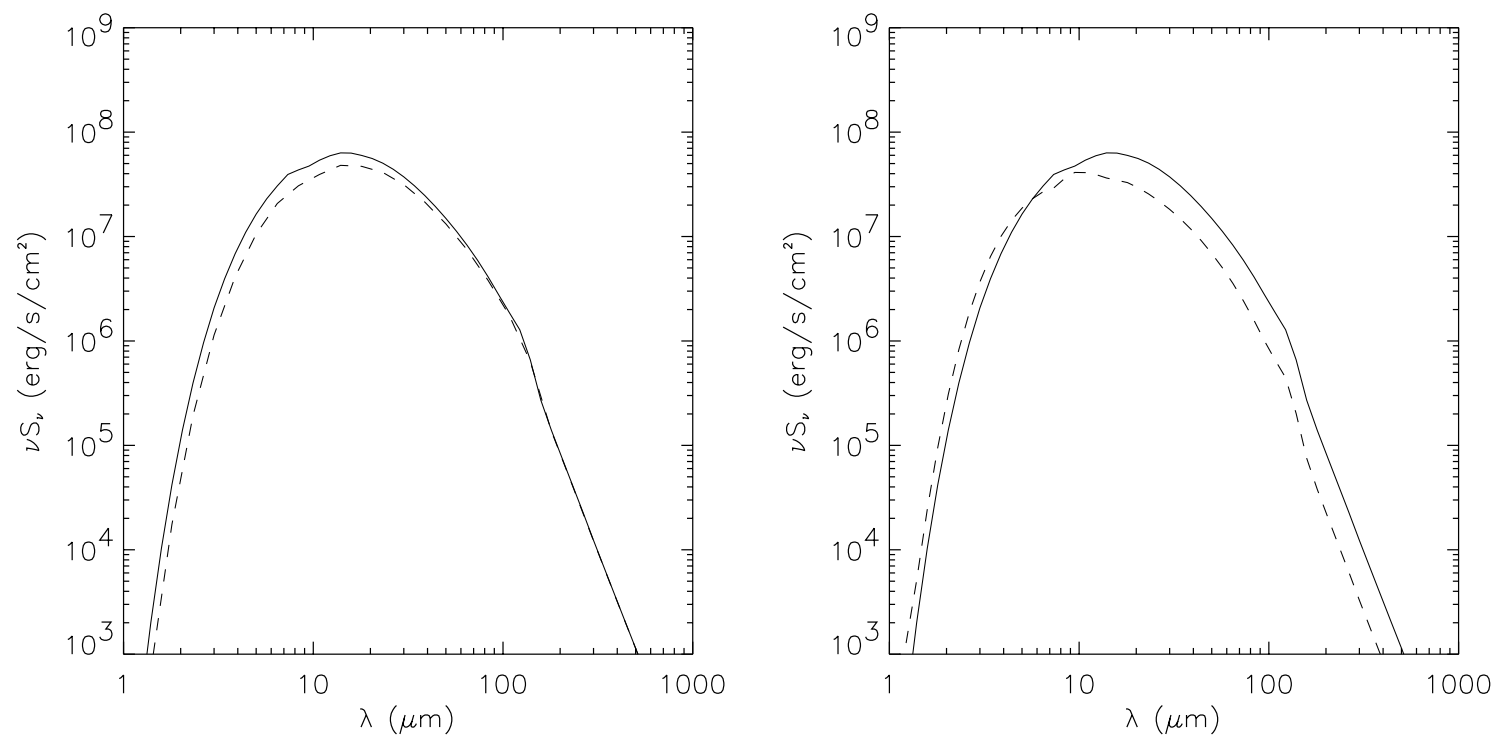

Fig. 4. Left panel: reference model compared with a model that has lower frequency resolution (dashed line). Right panel: comparison of the reference model ( $h_{0}=3 \mathrm{pc}$, solid line) with a model that has a lower value for the inner scale height $\left(h_{0}=1 \mathrm{pc}\right.$, dashed line).

Short Characteristics (see Dullemond \& Turolla 2000, for details), the radiation field at every location in the disk is computed as a function of frequency $v$ and direction $\mu, \phi$ of the radiation.

From this radiation field the mean intensity $J_{v}$ is computed:

$J_{v} \equiv \frac{1}{2} \int I_{v} \mathrm{~d} \Omega$

where $d \Omega$ is the solid angle. The dust temperature at every point can then be found by solving the equation:

$\int_{0}^{\infty} B_{v}(T) \mathrm{d} v=\int_{0}^{\infty} J_{v} \mathrm{~d} v$.

Once this (new) temperature structure is found, it is compared to the old one. If the relative difference is larger than 0.001 , we go back to the integration of the transfer equation (Eq. (1)), and keep iterating until the relative successive difference drops below the convergence criterion of 0.001 . We tested the convergence by starting the model with a cold $(1 \mathrm{~K})$ and a hot $(1500 \mathrm{~K})$ disk and comparing the resulting spectra. The results are indistinguishable, which indicates that the models are fully converged.

\subsection{Computing the spectrum}

The spectrum is obtained from the density and temperature distribution using a ray-tracing algorithm. For each discrete frequency an image of the torus is produced. These images are then integrated to obtain the spectrum $F_{v}$. To make sure that the pixels of these images accurately sample all relevant scales in the system, we distribute the pixels of the image not in a rectangle (as in normal digital images), but in concentric circles around the central source. Each circle contains 64 pixels, and the circles themselves have radii such than for every radial gridpoint on the computational domain there are two circles in the image. In this way there will be an equal number of pixelcircles per logarithmic interval in radius, and this guarantees that both the very inner parts of the disk (few pc) as well as the far outer parts (tens to hundreds of pc) will be sampled properly.

The spectra can be computed at any requested inclination. We select inclinations $(\theta)$ of 5, 30, 50, 60, 70 and 90 degrees. For small $\theta$ the inclination cannot be smaller than 0.1 radian, due to limitations of the code, so its lowest value is 5.7 degrees. All 2D/3D effects are included. For edge-on inclination $\left(\theta=90^{\circ}\right)$ one will see only the outer edge of the disk, while at more face-on inclination (say $\theta=5^{\circ}$ ) one will also see the central source and the warm inner regions of the disk. The angle at which the inner regions are shielded from view depends on the opening angle of the torus and the optical depth in our models.

\subsection{Flux conservation and resolution tests}

In the ideal situation, the torus will emit exactly the same amount of luminosity as it has absorbed from the central source. This requirement of flux conservation is a powerful check on the results of the code, and this check is in fact always performed by RADICAL at the end of each run. Moreover, RADICAL is built in such a way that radiation in optically thin areas of the system does not loose or gain flux through numerical diffusion. Unfortunately, in areas of large optical depth, flux conservation is not intrinsically guaranteed by the code, and occasionally the flux can be slightly over-predicted. In most cases we have energy conservation to within about $10 \%$, but sometimes (larger disk and/or high opacities) this error can go up to $20 \%$. Refinement of the grid and fine-tuning of the boundaries of the computational domain usually help to reduce this error, but there are feasibility limits to the number of grid-points that can be used. In this paper all models stay within this $20 \%$ tolerance, and most of the models in fact stay within $\sim 10 \%$.

The frequency resolution has to be high enough to prevent flux losses or overshoots. In Fig. 4 the effect of a lower 

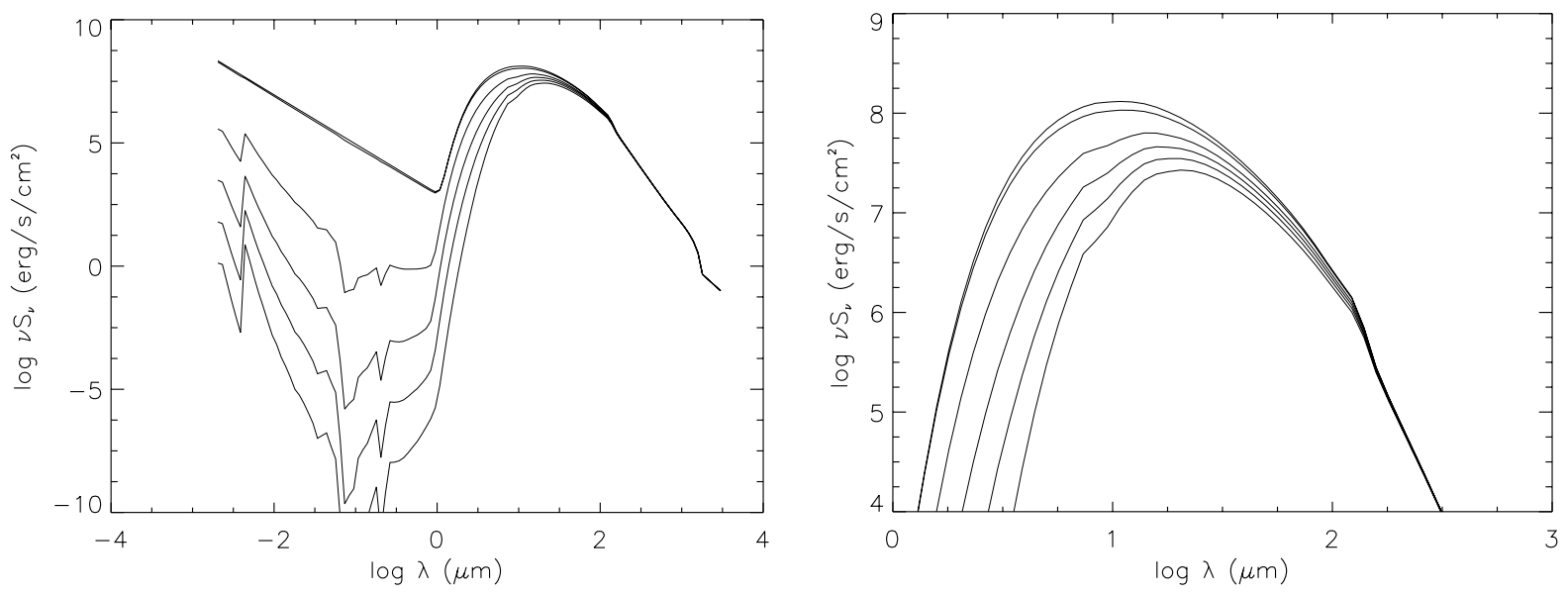

Fig. 5. The reference model, using $q=-2, \alpha=1, \beta=0, \tau_{20}=10$, with a disk size from 3 to $30 \mathrm{pc}$. Different lines are for different aspect angles, the top line corresponding to $\theta=5^{\circ}$, next are $30,50,60,70$ and $90^{\circ}$. In the right panel a zoom on the infrared regime is displayed.

frequency resolution is shown: with half the number of frequency points the flux deviates less than a factor of two. A higher frequency resolution does not significantly change the results.

Changing the scale height at the inner radius also affects the output spectrum. As shown in Fig. 4, decreasing $h_{0}$ mainly results in a lower output flux in the far-infrared. Since the disk is thinner, there is less cool dust in the disk. The decrease of flux is proportional to the decrease in $h_{0}$. It only marginally affects the shape of the spectrum, therefore it can be used to tune the results to observations for individual objects. When comparing colours, the assumed value of $h_{0}$ is irrelevant.

\subsection{Improvement on previous methods}

The large parameter space probed by our models is unique and allows for a full comparison of observations with these models. The dependence of the output spectrum on disk parameters has not been studied in this much detail before. For simplicity we have chosen to deal with a smooth dust distribution, before assessing the effects of a clumpy medium. One of the main problems has always been to reproduce the broad infrared SED observed in active galaxies: the effects of the geometry of the torus, its density, size, or opacity are not clear. We compare the models to available broad band data in order to assess the width of the resulting spectrum. Instead of fitting a large sample of objects, we will compare the colours of our models to the observed colours. Only for Cygnus A, a powerful nearby radio galaxy, will we study the actual fit in detail.

A second important issue has been the occurence of $10 \mu \mathrm{m}$ emission at small inclinations in most literature models, which is not observed. Although our frequency resolution does not allow an in depth study of the behaviour of the $10 \mu \mathrm{m}$ feature, we will discuss the effects of altering the grain size distribution on our models. The powerful radio-loud active galaxies lack proper observations of the $10 \mu \mathrm{m}$ region, and since we mainly aim to understand these objects, no direct comparison to infrared data will be presented in this paper. In a consecutive paper, high resolution models will be discussed, allowing for a direct comparison with new observations from the SIRTF satellite for a range of active galaxy types.

\section{Results}

In order to understand the effect of the different variables, we have defined a reference model, to which all other models are compared. The relevant parameters are given in Table 1. The reference model is chosen to be a conical disk with constant surface density, a size from 3 to $30 \mathrm{pc}$ and optical depth of 10 . This will allow a direct comparison to literature models, which generally have similar characteristics. However, we will use $q=-2.0$, which does introduce a different behaviour when considering the $10 \mu \mathrm{m}$ silicate feature, but which is a more realistic value when considering active galaxies. The optical depth of 10 is chosen to account for observed differences at $40 \mu \mathrm{m}$ restframe wavelength in quasars and radio galaxies (Heckman et al. 1992; van Bemmel et al. 1998, 2000). The size of the disk corresponds to sizes found in Seyfert galaxies, although it may be significantly larger in powerful radio galaxies.

\subsection{The reference model}

The model spectrum is shown in Fig. 5. The spectrum peaks between 10 and $20 \mu \mathrm{m}$, depending on inclination. For the different inclinations there are clear flux differences with inclinations (anisotropies) up to $100 \mu \mathrm{m}$. The knee at $110 \mu \mathrm{m}$ is a dust opacity feature. There is a slight indication of $10 \mu \mathrm{m}$ silicate absorption at larger angles.

The temperature and density distribution for the reference model are depicted in Fig. 6. The temperature in the disk is lower than in its optically thin surroundings, which is to be expected. In the inner regions the temperature drops steeply with radius, flattening at larger radii. Within the disk, the temperature in angular direction is more or less constant, with a steep increase at the edges to reach the temperature of the surrounding medium.

Below is described what the effect of varying the different parameters has on the model results, which is shown in Figs. $7-$ 9. These figures are a concise representation of the effects of changing the parameters. A complete set of hundreds of figures has been used to study the effect of the variables on anisotropy and $10 \mu \mathrm{m}$ absorption in the models. 

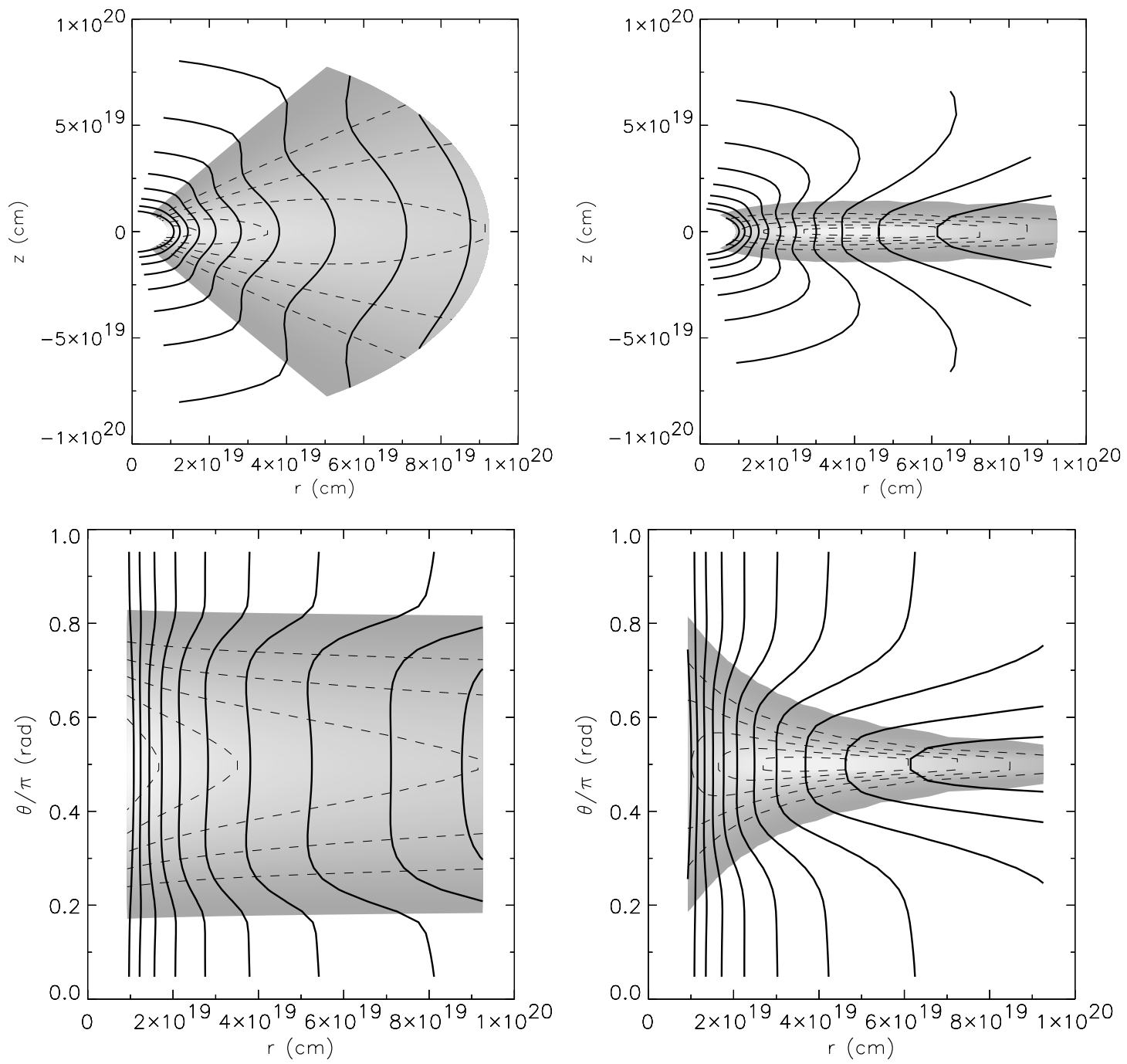

Fig. 6. The temperature and density distribution for the conical disk (left two panels) and the pillbox disk (right two panels). Upper panels are in $z$ coordinates, lower panels in $\theta / \pi$. Grey scale shows the density distribution of the dust, the solid lines are temperature contours, corresponding to $600 \mathrm{~K}$ (leftmost) and decreasing with steps of $50 \mathrm{~K}$.

The depth of the $10 \mu \mathrm{m}$ absorption shown in Fig. 9 is determined using a similar method as Pier \& Krolik (1992): a powerlaw is fitted through the 6.5 and $13.9 \mu \mathrm{m}$ points. However, since the model curves have a grey-body shape, fitting a power-law will mimick silicate emission where none is present. This is circumvented by adding to the power-law fits the residual from the model with $5^{\circ}$ inclination, where no silicate emission or absorption occurs, resulting in a continuum that approaches a grey-body shape in the region 5-15 $\mu \mathrm{m}$. Subsequently, the difference between the continuum and the $9.7 \mu \mathrm{m}$ gridpoint is measured. The depth $\tau_{9.7 \mu \mathrm{m}}$ is positive for aborption.

- $\alpha$ : changing the physical shape of the disk to a pillbox $(\alpha=0)$ or flaring disk $(\alpha=9 / 7)$. The flaring disk and conical disk have similar spectra, slightly varying with inclination. The pillbox disk is less luminous at all inclinations, but this is more obvious at smaller angles. It is hotter than the reference model, with the peak at shorter wavelengths for smaller inclinations. The wavelength of the emission peak does not shift significantly with inclination, but there is an indication of increased anisotropy. The flared models have a higher degree of isotropy than the reference model, but show a similar shift in the peak wavelength with inclination. They also show a hint of $10 \mu \mathrm{m}$ absorption at small inclinations, but this effect depends strongly on the optical depth and the size of the disk.

- $\beta$ : changing the surface density from a constant to a more centrally concentrated configuration $(\beta=-2)$. For fixed $\alpha$, the models differ slightly at large inclinations, and marginally at small inclinations. The more centrally concentrated, the more the spectrum moves to shorter wavelengths. At large $\theta$, there is a hint of $10 \mu \mathrm{m}$ absorption, which deepens when $\beta$ changes from 0 to -2 . The depth does not depend on the value of $\tau_{20}$. The $10 \mu \mathrm{m}$ absorption is absent in the $30-300 \mathrm{pc}$ disk.

- $\tau_{20}$ : changing the optical depth at $20 \mu \mathrm{m}$ from 5 to 50 . The higher opacity models have broader infrared spectra and shorter peak wavelengths at small angles. For larger inclination the high opacity models are not much broader, but clearly have more far-infrared emission. The peak 

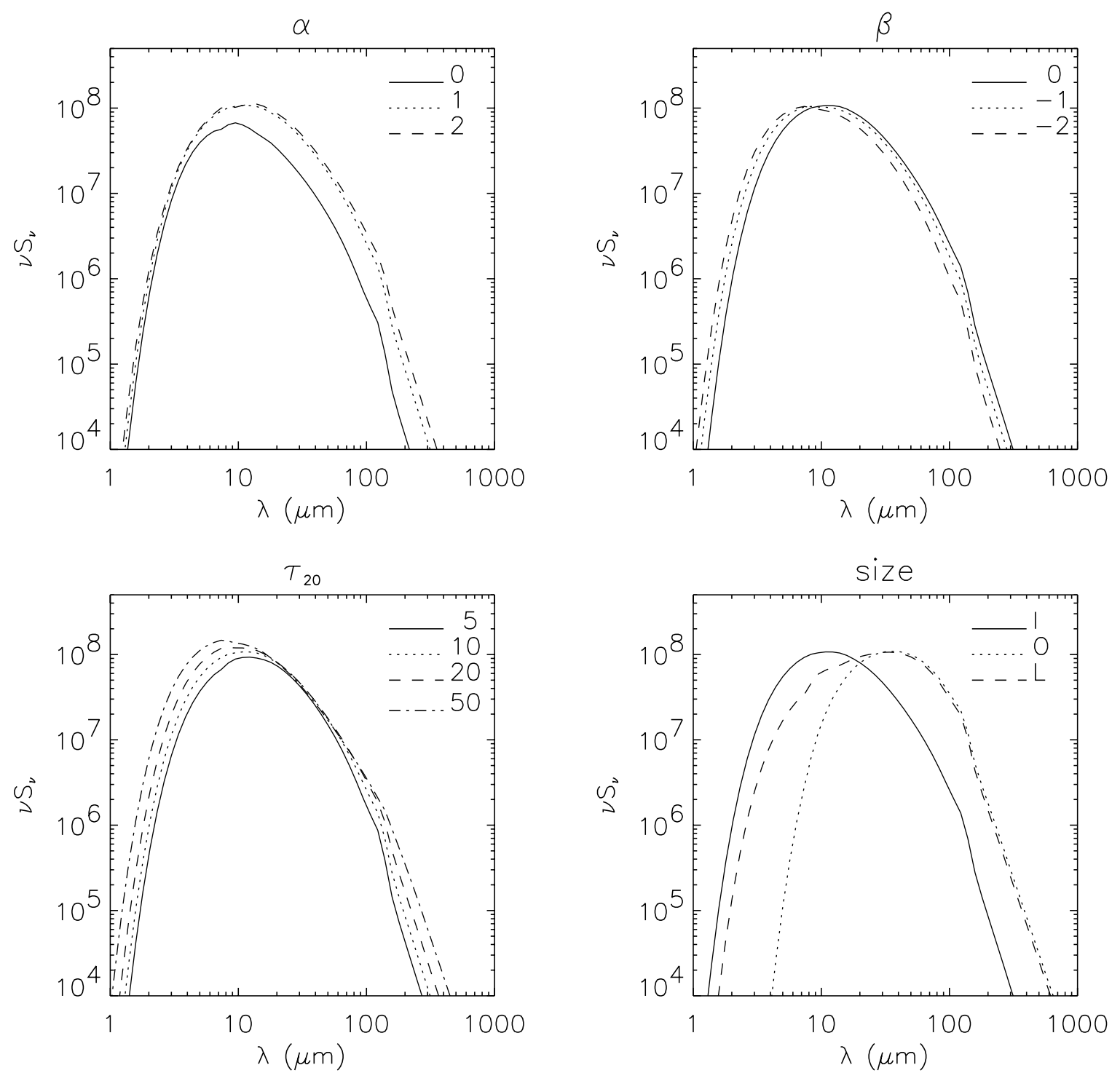

Fig. 7. Comparison of the models with different varying parameters for a fixed inclination of $\theta=30^{\circ}$. The non-varying parameters have the values as for the reference model: $\alpha=1, \beta=0, \tau_{20}=10$ and size $3-30 \mathrm{pc}$.

wavelength hardly changes at larger angles. An important effect is the increase of anisotropy with increasing $\tau_{20}$, but the wavelength at which the dust becomes optically thin does not vary significantly. There is a hint of $10 \mu \mathrm{m}$ absorption in the low opacity models at large inclination.

- size: the outer disk emits at much longer wavelengths than the inner disk, and shows a stronger anisotropy at longer wavelengths. The large disk has a much broader spectrum at small angles, indicating a larger temperature range. At large inclinations the outer and the large disk are almost identical. The large disk has clear $10 \mu \mathrm{m}$ absorption. A hint of this is seen in the inner and outer disk.

- $q$ : using Galactic dust instead of a large-grain dominated mixture. For larger inclinations the $10 \mu \mathrm{m}$ absorption becomes deeper. There is a hint of $10 \mu \mathrm{m}$ emission, which strongly depends on the optical depth and disk size. For smaller optical depth, the emission is clearly present. For larger optical depth, the absorption is only seen in intermediate inclinations. A direct comparison of $\tau_{9.7 \mu \mathrm{m}}$ shows minor silicate emission at inclinations around $30^{\circ}$, but up to a factor of 5 increase of silicate absorption at angles larger than $40^{\circ}$. In the outer disk, the absorption is deeper at larger inclinations, but there is a clear emission feature for small angles. The anisotropy is less pronounced than in the reference model, although it is visible up to $100 \mu \mathrm{m}$ as well. The peak wavelength is unchanged.

It is illuminating to examine the spatial distribution of the emission in the disk. In Fig. 10 we show images of the reference model, at 30 and $200 \mu \mathrm{m}$, and with inclination of 30 and $70^{\circ}$. The images display the entire angular grid, causing them to have a circular cut-off, independent of the inclination of the disk. At $30 \mu \mathrm{m}$ the disk is optically thick and only the inner regions radiate. At $70^{\circ}$ the inner regions are not seen directly, but some emission is seen from the opposite inner side of the torus. At $200 \mu \mathrm{m}$ the dust is optically thin and the entire disk will radiate. At $30^{\circ}$ the outermost regions are not as bright as the rest, since they are somewhat cooler. At $70^{\circ}$ the inner 

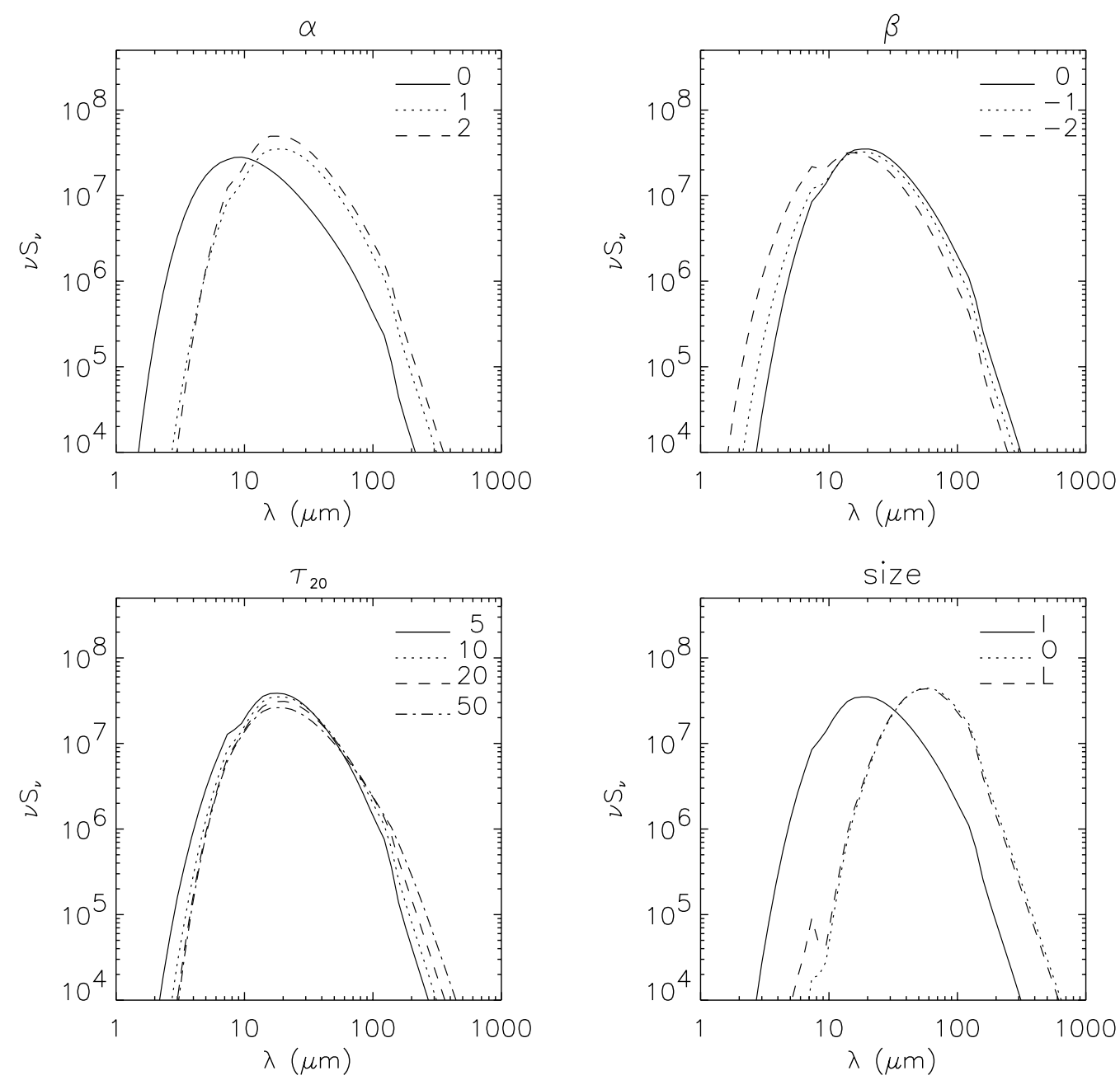

Fig. 8. As Fig. 7, but for inclination angle $\theta=70^{\circ}$.

regions are now seen direct, since the dust is transparent. They are the brightest part of the disk, since this is where the column density is highest.

The effect of varying the parameters is shown in Fig. 11. The top row shows again the reference model. The second row shows the pillbox models ( $\alpha=0)$, which are clearly less bright. This difference is most pronounced at $200 \mu \mathrm{m}$. They are of course flatter at $70^{\circ}$. The third row displays the $\beta=-2$ models, i.e. models with equal mass per logarithmic interval. Here the inner regions dominate all the images, since they represent the densest parts of the disk. This is especially clear in the $200 \mu \mathrm{m}$ images, where the disk is optically thin. The final row contains the images for a disk with $\tau_{20}=50$. At $30 \mu \mathrm{m}$ the emission is more centrally concentrated and the contrast with the outer regions is larger than for the reference model. However, for the $200 \mu \mathrm{m}$ images the opacity is irrelevant, and the images are comparable.

\subsection{Comparison with models in literature}

The resulting spectrum from our reference model can be compared to literature models with comparable disk and dust parameters. The main difference between our model and the literature models is the absence of significant $10 \mu \mathrm{m}$ emission at small inclinations, and only weak $10 \mu \mathrm{m}$ absorption at larger inclinations. However, since all literature models use Standard Galactic Dust, a comparison at $10 \mu \mathrm{m}$ is unrealistic.

A qualitative comparison of our reference model with literature models with comparable parameters reveils that the general shape of our output spectrum is best matched by the Pier \& Krolik (1992) models (hereafter PK92). The models by Granato \& Danese (1994) (hereafter GD94) also match the shape quite well, but the Efstathiou \& Rowan-Robinson (1995) (hereafter ER95) models are very different, with a much flatter SED in the mid-infrared and often a peak in the nearinfrared. Our reference model becomes optically thin longward of $100 \mu \mathrm{m}$. Only the PK92 models match this, other models all become optically thin between 60 and $80 \mu \mathrm{m}$. In all literature models changing the inclination causes a continous decrease in flux at a fixed wavelength. The GD94 models show a very discrete change in flux between 45 and 75 degrees inlination. As a consequence, there is no obvious difference between their models with 0 and 45 degrees inclination, and neither between the models with inclinations of 75 and 90 degrees, except for the very thick and compact geometries.

For a more quatitative comparison we have calculated an anisotropy value, defined by taking the flux ratio for face-on 

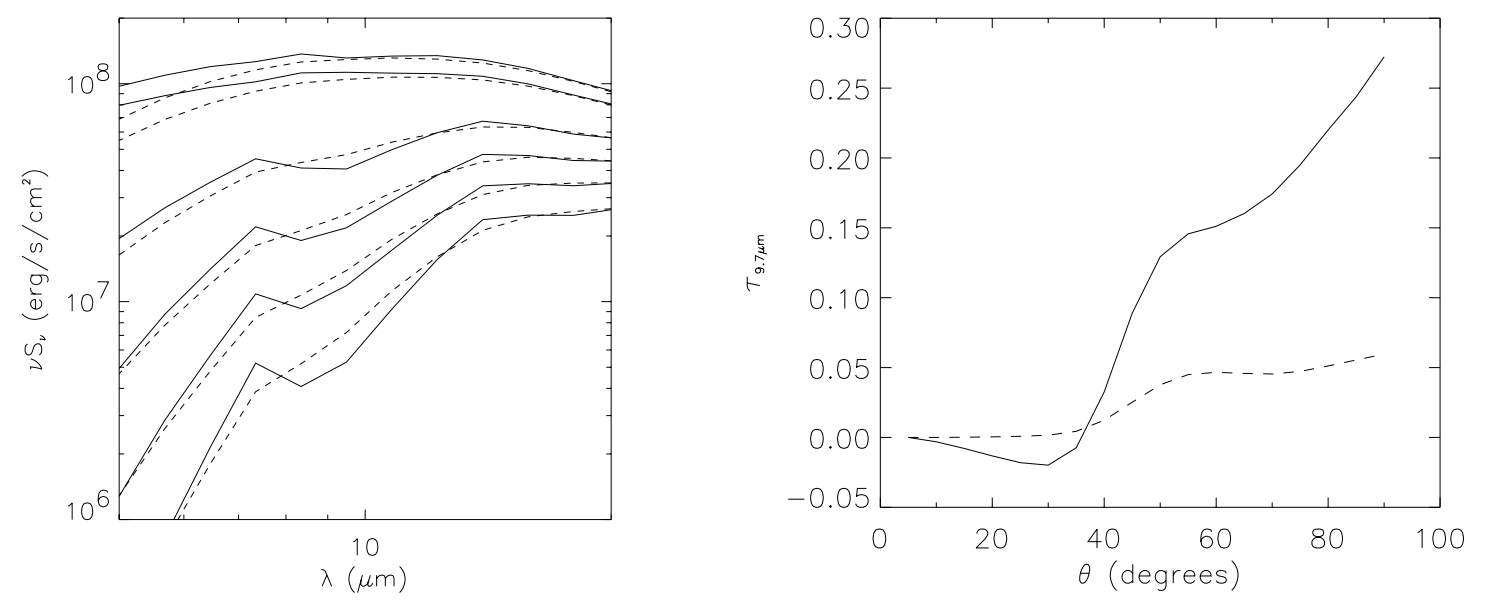

Fig. 9. Comparison of the $10 \mu \mathrm{m}$ silicate absorption in the reference model when using different opacity laws. The solid line represents MRN dust $(q=-3.5$, Galactic), the dashed line the larger grain dust $(q=-2)$ adopted in this paper. Left panel: a zoom on the spectrum for inclinations $5^{\circ}, 30^{\circ}, 50^{\circ}, 60^{\circ}, 70^{\circ}$ and $90^{\circ}$ (from top to bottom). Right panel: depth of the $10 \mu \mathrm{m}$ absorption feature with inclination (positive values indicate absorption).

over edge-on oriented disks at $20 \mu \mathrm{m}$. For the literature models that have parameters closest to our reference model, we find this ratio to be $\sim 5$. This is consistent with the anisotropy value in our reference model.

Clumpy torus models, as described by Nenkova et al. (2002) are in general flatter than our reference model, which is especially prominent at small inclinations. Their anisotropy value is also $\sim 5$, as in our reference model, and they do not become optically thin shortward of $100 \mu \mathrm{m}$. Depending on the mean free path of the clumps, the models have a more pronounced peak around $60 \mu \mathrm{m}$, and become more luminous.

A more detailed comparison is not feasible, since the models in literature use different setups and calculation methods. All observed differences between our results and literature models can be ascribed to this.

\section{Understanding the behaviour}

The output of a dusty disk depends mainly on two properties: the temperature and the mass of the dust. If the dust is further away from the emission source, it will be cooler, and therefore emit at longer wavelengths. At shorter wavelengths the dust is self-shielding, since the dust is optically thick for its own radiation. The inner regions will become very hot, but these can only get rid of the energy at wavelengths where the dust is optically thin. As a consequence, the inner regions will be the brightest at the shorter wavelengths, and only in the far-infrared will the entire disk start to radiate. This effect is clearly seen in the images (Fig. 10).

In a pillbox model, the $\tau=1$ surface is "squeezed" more to the equatorial plane and, hence, compared to the other models less radiation of the central source is intercepted. Pillbox models are therefore less luminous. The difference between conical and flaring disks is less pronounced. Nevertheless, for a flaring disk, some dust at large radii will have a direct line of sight to the central source and be warmer. This effect is best observed at large inclinations (see Fig. 8).
When we vary $\beta$ from 0 to -2 , more mass will be concentrated in the inner regions. This dust will be hotter, and therefore brighter in the near- and mid-infrared. Since the dust is more centrally concentrated for $\beta=-2$, there is less mass in the outer regions, and therefore less cool dust, as seen in Fig. 11. As a consequence the far-infrared emission will decrease in flux, and the entire spectrum shifts to shorter wavelengths.

Increasing the optical depth increases the amount of absorbed radiation. For higher values of $\tau_{20}$ it will be more difficult to transport absorbed energy into the torus. As a consequence the inner parts get hotter, but the outer parts will remain cooler. This results in a wider temperature distribution, which translates into a broader energy distribution for small inclinations (Fig. 7). For large inclinations, the increased amount of cold dust is most important. Since the inner regions are shielded from direct view, the energy distribution shifts to longer wavelengths.

The distance to the central source is critical in the models. At large inclinations, we cannot view the inner edge of the torus direct. The hot dust is shielded from view by the cool outer regions. For this reason, the large disk and outer disk have comparable spectra. Overall the inner disk is much warmer and brighter at the shortest wavelengths. At small inclination, the whole disk is visible and both the inner and large disk, which have hot dust close to the central source, are much brighter at shorter wavelengths. The large disk and the outer disk show very similar behaviour at the longest wavelengths where the dust is optically thin. The large disk produces less near- and mid-infrared emission than the inner disk, even though its inner radius is similar, since the temperature gradient is steeper. This is also related to the model setup: since the disk is larger, a smaller value for $\Sigma_{0}$ is required to obtain the requested equatorial optical depth, and hence the total opacity is lower.

Assuming $q=-2$ instead of using Galactic dust mainly affects the $10 \mu \mathrm{m}$ silicate feature. Although our models clearly lack significant silicate emission, they still display clear absorption, albeit not as deep as the Galactic dust models. 

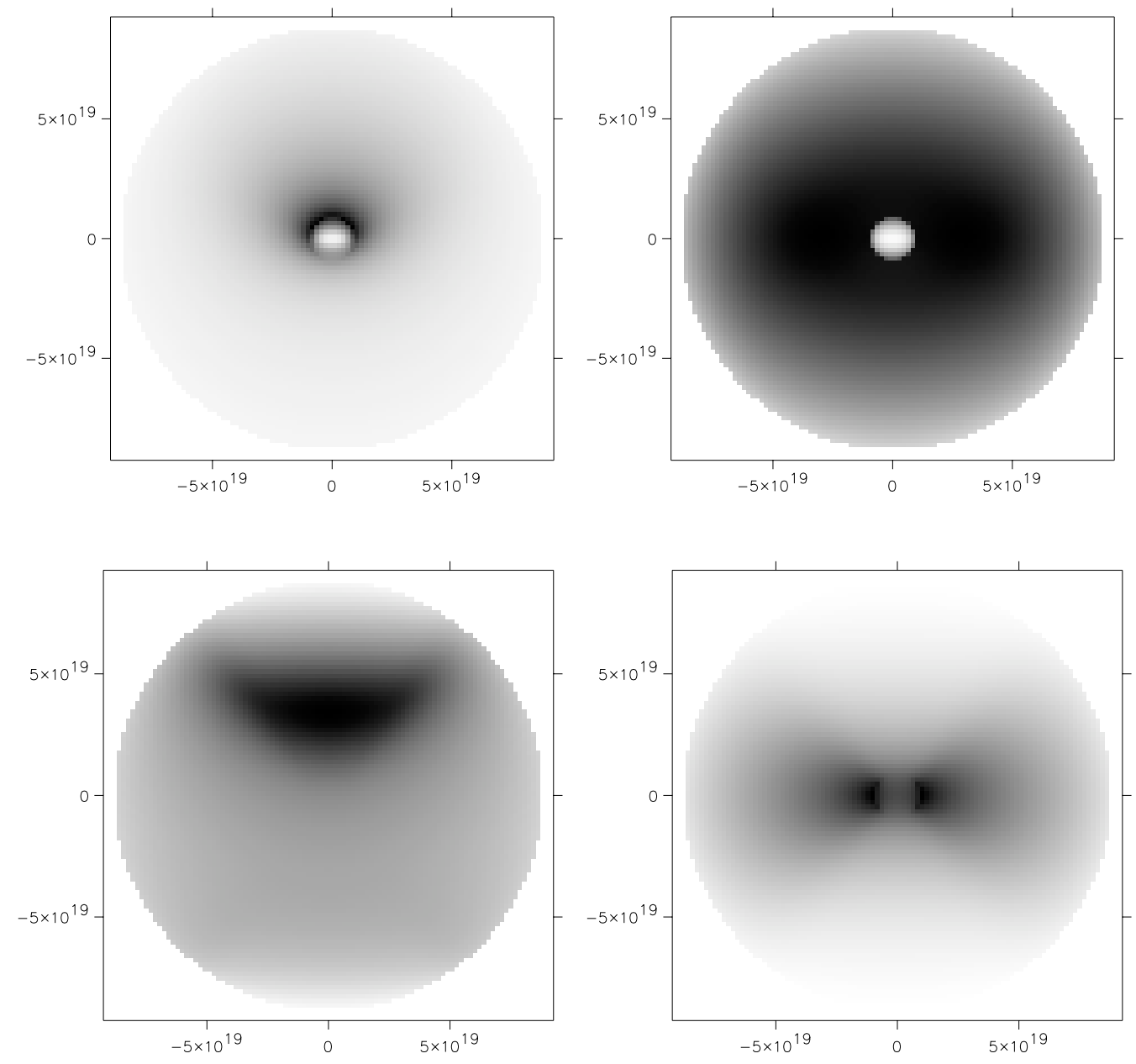

Fig. 10. Images of the reference model, using a circular aperture. Top panels are for $30^{\circ}$ inclination, bottom panels for $70^{\circ}$. Left panels are the $30 \mu \mathrm{m}$ images, right panels are at $200 \mu \mathrm{m}$. The grey scale is inverted: dark areas represent more emission. The central white region is where the disk ends and the central emission source is located. The outer circle represents the aperture.

The detailed behaviour of the silicate feature will be the purpose of future high frequency resolution models.

\section{Comparing the models to observations}

\subsection{Colour comparison}

Using the known infrared colours of samples of active galaxies, we can attempt to constrain the parameters of the obscuring medium. We will use the IRAS bands $(12,25,60$ and $100 \mu \mathrm{m})$ which are also available as ISO bands. We have selected all 3C radio-loud active galaxies from the literature (Golombek et al. 1988; Neugebauer et al. 1986) with a least three solid detections in three of the IRAS bands, and with small redshifts $(z<0.5)$. It should be mentioned, however, that IRAS detected only a few radio-loud active galaxies, which all lie close to the detection limits of the instrument. In addition we selected a sample of starburst, ultra-luminous and Seyfert galaxies with four IRAS detections from literature (Sekiguchi 1987; MacKenty 1989).

For all radio-loud objects we assured that the infrared emission is of thermal nature. From Heckman et al. (1992) we have constructed the colours for samples of quasars and radio galaxies. All data are overplotted on the models, while varying different parameters in Figs. 12-14. The $\alpha$ symbol on the axis, annotated with two wavelengths, represents the spectral index or colour, defined as $F \propto v^{\alpha}$, while the $\alpha$ symbol in the plots, without annotation, refers to the geometry of the disk. We will refer to the colours as hot $\left(\alpha_{12}^{25}\right)$, warm $\left(\alpha_{25}^{60}\right)$, and cold $\left(\alpha_{60}^{100}\right)$. For an increase in spectral index the flux at the shorter wavelength increases with respect to the flux at the longer wavelength. Hence an increase in the value for the colour implies more flux at shorter wavelength.

The objects within a given class cluster together. The quasars have a narrow range of hot and cold colours, both lie between 0 and -1 . The warm colour ranges from 0.3 to -2 . The narrow-line radio galaxies have cooler hot colours than the quasars, ranging from -0.5 to -2 . The warm colours show approximately the same values, from 0 to -1.5 , but the cold colour has a large range from 1 to almost -2 . For the broadline radio galaxies the hot colour is comparable to that of the quasars, but the warm colour is clearly warmer, having typical values between 1 and -0.5 . The cold colour is only determined for one object, the rest lack $100 \mu \mathrm{m}$ detections. The starburst and ultra-luminous galaxies lie clearly in a different region than the active galaxies in the hot vs. warm plot, while their cold 

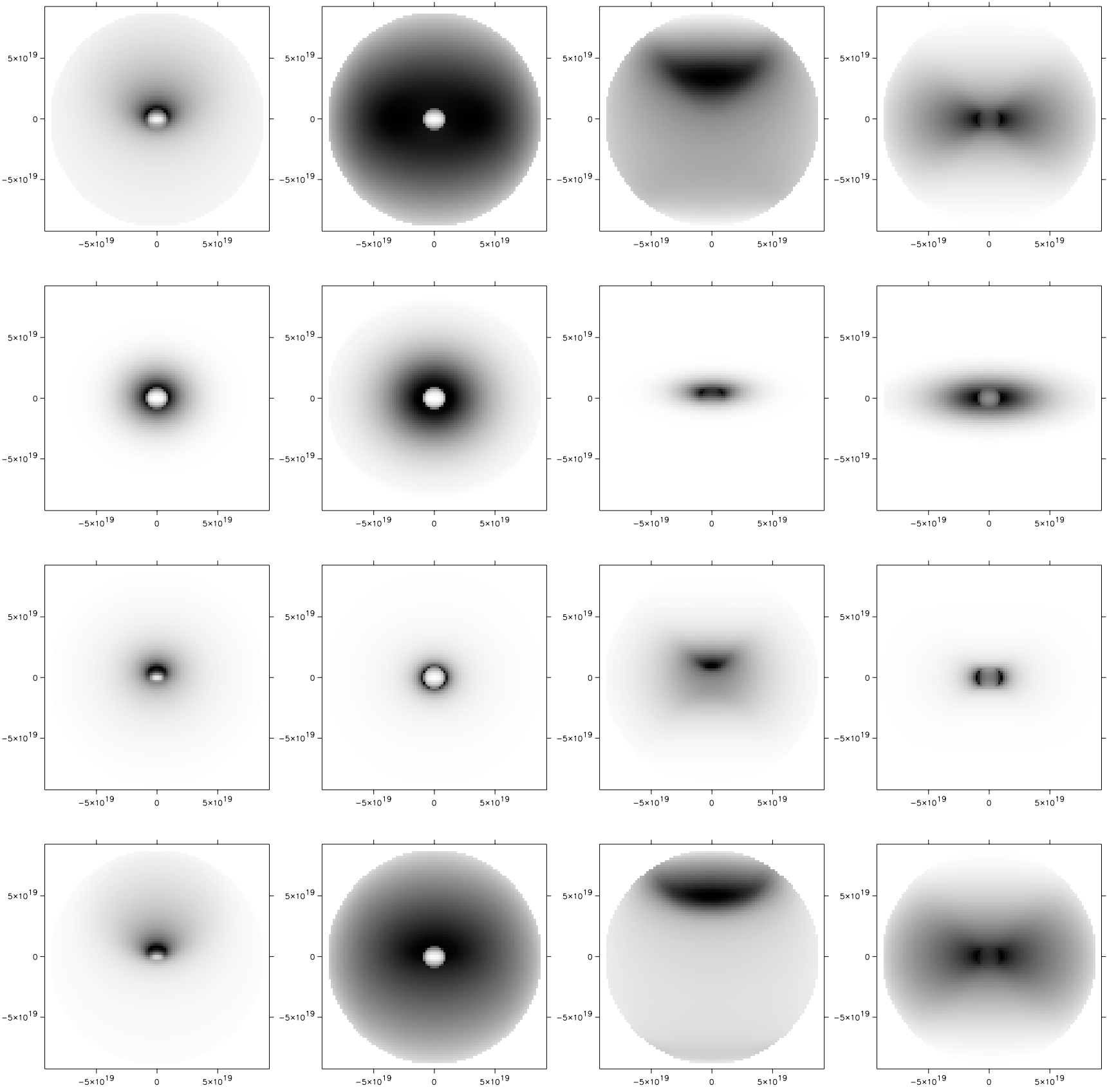

Fig. 11. Images of the disk when varying the different parameters. The top row is the reference model, then from top to bottom per row are changed: $\alpha=0, \beta=-2$ and $\tau_{20}=50$. From left to right the images are for $30^{\circ}$ at 30 and $200 \mu \mathrm{m}$, and $70^{\circ}$ at 30 and $200 \mu \mathrm{m}$ respectively.

colours are comparable to the cold colours of the radio-loud active galaxies. The Seyfert 1 colour distribution is indistinguishable from the colours for the radio-loud active galaxies, but the Seyfert 2 galaxies overlap both the active galaxies and the starbursts in their hot and warm colours.

There are no objects which have a positive hot colour in the entire sample, and only a few which have a positive warm colour. Nevertheless, the colours of the three radio-loud classes do not separate out too much. From an infrared point of view, these three classes are a somewhat arbitrary classification of a continuum distribution of types.

\subsubsection{Models with $\beta=0$ and $\tau_{20}=10$}

In Fig. 12, we have fixed $\beta$ and $\tau_{20}$, and varied the disk size (3 different lines in plot), $\alpha$ (the different plots) and the orientation (along the lines, edge-on to face-on is from left to right). The overplotted data are for objects which are presumed to represent a continuum of different orientations, from quasars to narrow-line radio galaxies. The filled symbols are for ensembles of quasars and radio galaxies.

Changing $\alpha$ from $\alpha=0$ (pillbox) to $\alpha=1$ (conical) has a profound effect on the colours of the disk. A further increase to $\alpha=2$ (flaring disk) has little effect. These effects are in line with the SEDs shown in Figs. 7 and 8. 

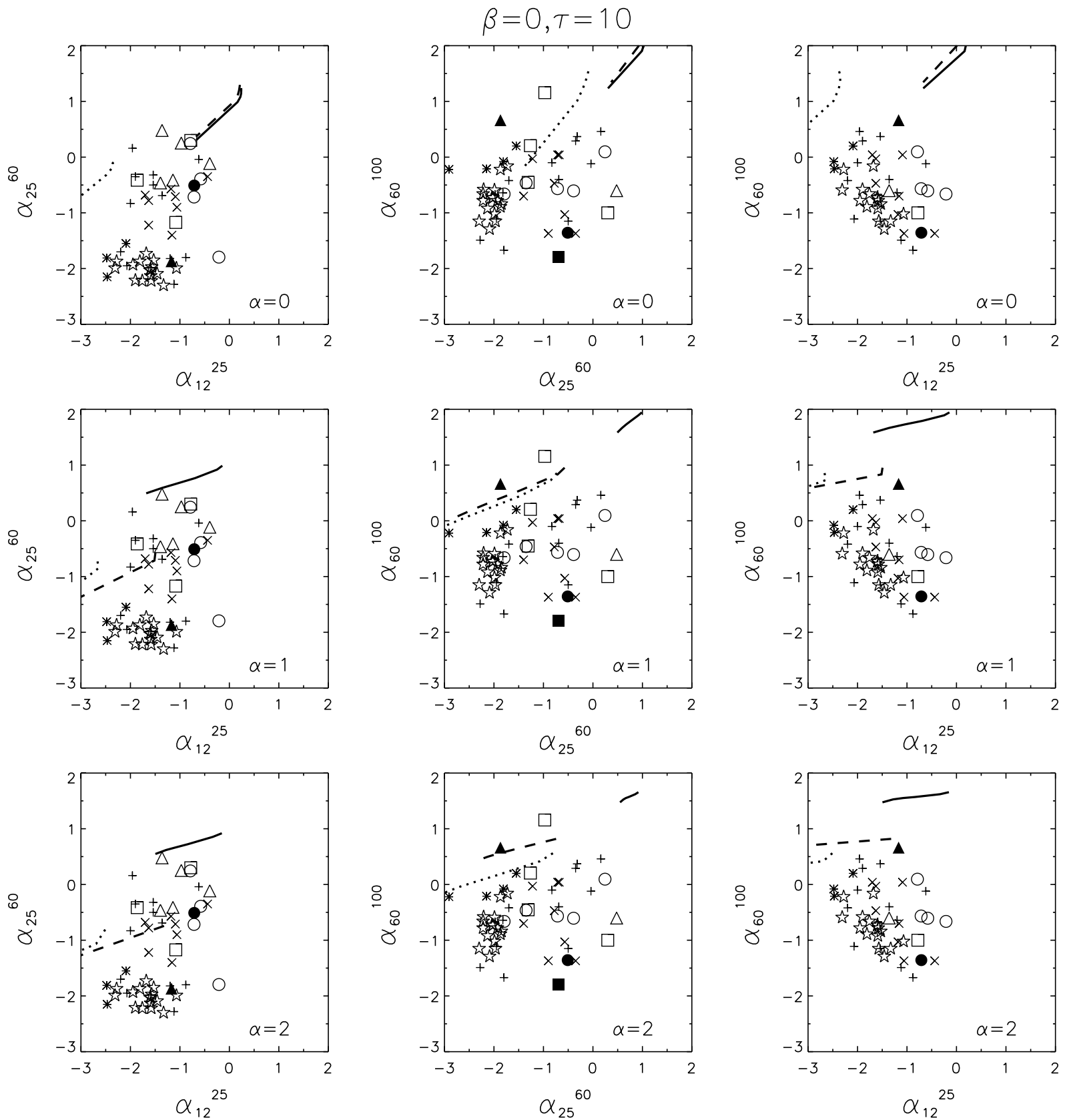

Fig. 12. Colour-colour plots for models with $\beta=0, \tau_{20}=10$ and varying $\alpha$ and disk size. Lines indicate varying inclination: from left to right corresponds to edge-on to face-on orientations. Solid lines are for the inner disk, dotted for outer disk, dashed for large disk. The objects are coded as follows: open circles = quasars, open squares = narrow-line radio galaxies, open triangles = broad-line radio galaxies, filled circle $=$ Heckman quasar sample, filled square $=$ Heckman radio galaxy sample, filled triangle $=$ Cygnus $\mathrm{A}$, crosses $=$ Seyfert 1 , plusses $=$ Seyfert 2 , asterisks $=$ ultra-luminous galaxies, open stars $=$ starburst galaxies .

\subsubsection{Models with $\alpha=1$ and $\tau_{20}=10$}

In Fig. 13, the value for $\alpha$ is fixed, as is $\tau_{20}=10$. Decreasing $\beta$ to -2 the models move up, except the outer disk, indicating more positive warm and cold spectral indices. The hot colour only increases with decreasing $\beta$ in the large disk.

\subsubsection{Models with $\alpha=1$ and $\beta=0$}

So far we have fixed the value of $\tau_{20}$, but this can of course also vary. In Fig. 14 we have fixed $\alpha=1$ and $\beta=0$ and varied $\tau_{20}$ in the different plots. Along the lines we still vary the inclination. Increasing $\tau_{20}$ will widen the range of the hot and warm colours of the models with changing the inclination. The warm colour also becomes cooler with increasing opacity. The cold colours have approximately the same range when varying $\tau_{20}$, but become cooler for higher values.

\subsubsection{Comparison with observations}

As judged from the plots, for all models the cold colours appear inconsistent with the observations. This implies that either the 

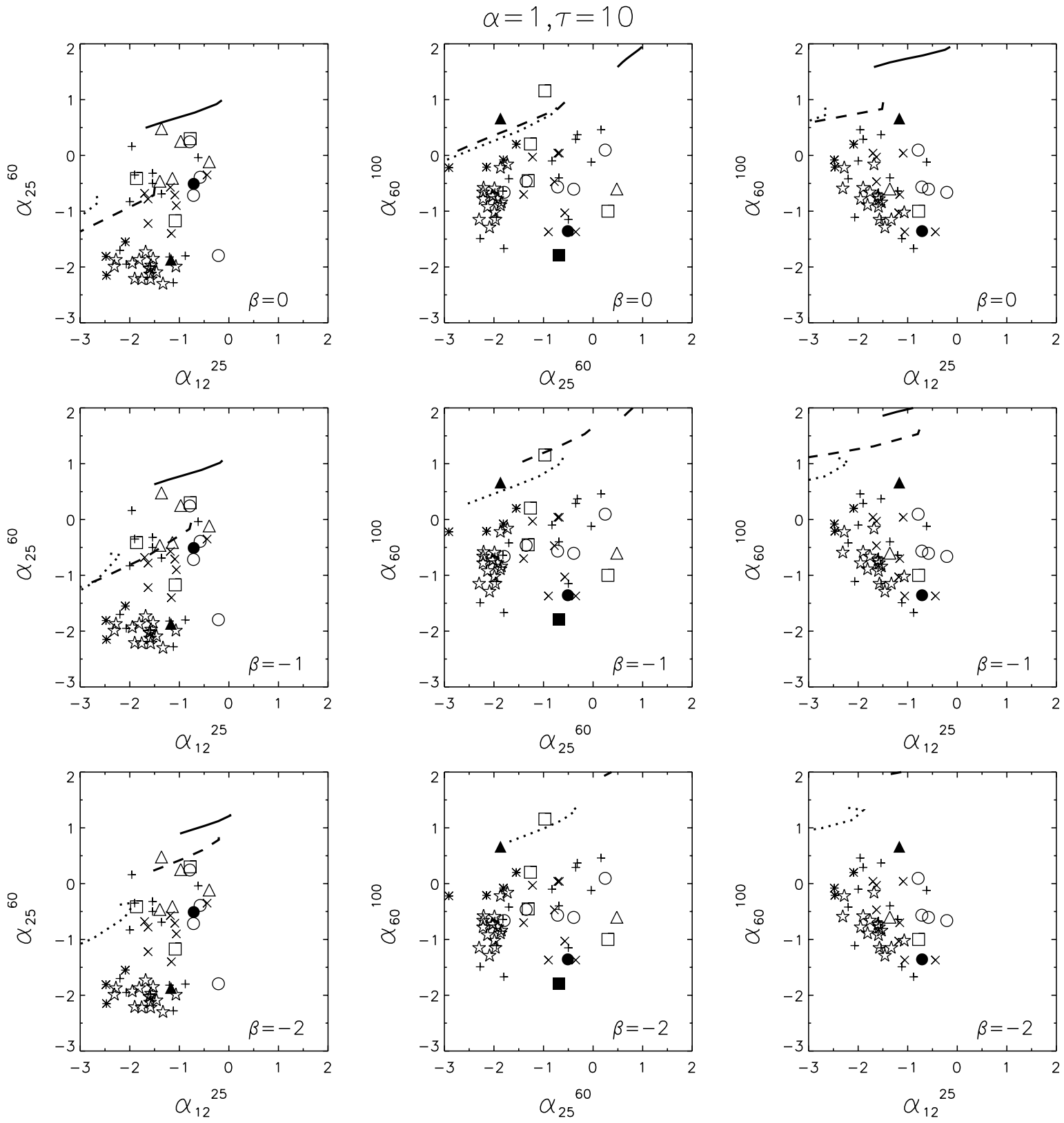

Fig. 13. Colour-colour plots for models with $\alpha=1, \tau_{20}=10$ and varying $\beta$ and the disk size. Lines and symbols as in Fig. 12 .

observed $60 \mu \mathrm{m}$ flux or the $100 \mu \mathrm{m}$ flux, or both are not fitted by the models. The hot colours in the models span the same range as the observed colours for active galaxies, but in the hot vs. warm colour plot, the warm colours in the models do not cover the entire range of observed values. This indicates that already at $60 \mu \mathrm{m}$ the spectrum starts to deviate. Hence, both the 60 and $100 \mu \mathrm{m}$ emission in the active galaxies are dominated by a different component.

At shorter wavelengths, the difference in hot and warm colours between starbursts and active galaxies indicates that the 12 and $25 \mu \mathrm{m}$ emission in active galaxies arises from a component unrelated to star-formation, most likely the active nucleus. Longward of $60 \mu \mathrm{m}$ the colours of active galaxies are indistinguishable from the colours of non-active galaxies, which implies that the emission process is the same in both groups. This favours star-formation as the dominant process of far-infrared emission in active galaxies. A similar conclusion is reached by studies of infrared colours of Seyfert galaxies longward of $100 \mu \mathrm{m}$ by Spinoglio et al. (2002), who find that this emission is independent of the presence of an active nucleus. Furthermore, Prieto et al. (2001) conclude that coronal line strength is strongly correlated with near- and mid-infrared emission, but not at all with far-infrared, indicative of a different heating mechanism for the far-infrared emission.

In the hot vs. warm colour plot the narrow-line and broadline radio galaxies are well fitted by the models. For the other colour plots only a few narrow-line radio galaxies are still within the range of model colours. The outlying quasar in the hot vs. warm colours is $3 \mathrm{C} 48$, known to have a large starformation rate, and possible all its infrared emission arises in star-forming regions (Canalizo \& Stockton 2000). Its warm colour is comparable to the warm colour of objects with known 

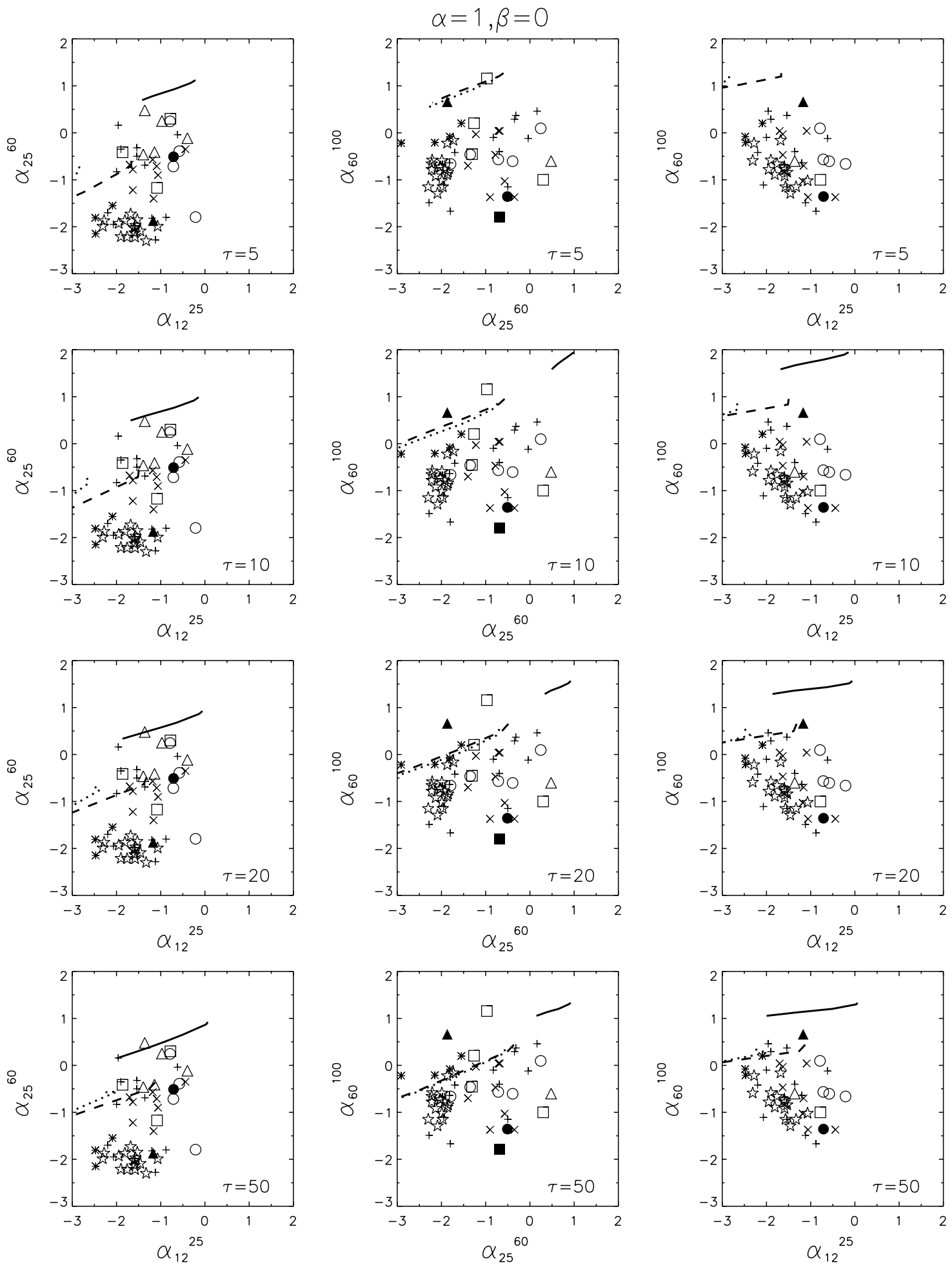

Fig. 14. Colour-colour plots for models with $\alpha=1, \beta=0$ and varying $\tau_{20}$ and disk size. Lines and symbols as in Fig. 12.

large star-formation rates, but its hot colour is clearly warmer, indicative of very hot dust. This indicates that a significant contribution from an active nucleus will cause objects to have a more positive warm colour, while their cold colours are indistinguishable from the colours of star-forming objects.

The inner disk does not fit the observed colours well, except in the hot vs. warm colour plot. The large and outer disk do better for $\alpha=1$ and $\alpha=2$ disks and large inclinations, but also fail in the hot vs. cold colours. The pillbox disks do not fit any of the observations. For decreasing $\beta$ all models move away from the observations. Increasing $\tau_{20}$ moves the models closer to the observed colours, but the discrepancy in cold colours is not explained. 
We note with interest that the ultra-luminous galaxies are well fitted by the high opacity models in the cold vs. hot and cold vs. warm colour plots. Also Cygnus A lies closer to the models than the remainder of the radio galaxy population. For the ultra-luminous galaxies it is known that starformation dominates the far-infrared output, and also Cygnus A is has strong evidence for significant star-formation in the host galaxy. Hence, star-formation can mimic the SED of an obscuring torus and its relative contribution must be assessed by other means, e.g. PAH emission, optical absorption lines, to fully disentangle the two infrared components

\subsubsection{Conclusions from colour-colour plots}

Most objects have negative cold spectral indices which are not explained by the models. This could very well be due to a contribution from other components, such as additional cold dust from star-formation regions. Observational evidence for a second dust component heated by stars in active galaxies is found in several studies (Prieto et al. 2001; Spinoglio et al. 2002; van Bemmel \& Barthel 2001). Therefore, we conclude that the torus emission only dominates the SED to about $25 \mu \mathrm{m}$, while the observed 60 and $100 \mu \mathrm{m}$ flux arise in a second infrared component.

With respect to the unification theory, there is no trend in the data for radio galaxies and Seyfert 2 to be more consistent with large inclination models, or quasars and Seyfert 1 with small inclinations. In the hot vs. warm plots all radio galaxies lie closer to the models than the quasars, while the objects with known large star-formation rates occupy a clearly distinct region, having cooler hot and warm colours. The broad-line radio galaxy colours are consistent with the lack of large scale cold dust, as discussed in van Bemmel \& Barthel (2001). If the torus is the only infrared component in broad-line radio galaxies, they should also be consistent with the models in their cold colours, but we lack detections at $100 \mu \mathrm{m}$ to confirm this. As emphasized above, the differences between models and observations are indicative of a second component in the far-infrared.

\subsection{Comparison with Cygnus $A$}

For individual objects, the model parameters can be constrained by a direct fit to the observed infrared spectrum. One of the best objects to test our models is the narrow-line radio galaxy Cygnus A, since it is the best observed radio-loud active galaxy in the infrared, and has a rather well determined central continuum. IRAS and ISO have observed Cygnus A in the infrared and additional (sub-)millimeter data are available to determine the non-thermal contribution. From optical observations the size of the obscuring torus has been constrained to lie between a few parsecs inner radius, up to maximum of a few hundred parsecs outer radius (Imanishi \& Ueno 2000). Also, we know that the angle of orientation of the radio jet is $\sim 50$ degrees (Carilli \& Barthel 1996), allowing us to vary $\theta$ around this value. For the models we fix $\alpha=1$ and $\beta=0$, since these are the best values from the colour-colour fitting. We use $q=-2$ for the dust grain size distribution.
We have constructed models normalized to the observed hard X-ray luminosity and spectral slope, and taken into account size limitations of the torus in Cygnus A. The inner and outer radius are varied with one extra value: $r_{0}=3,10$ and $30 \mathrm{pc}, r_{\text {out }}=30,100$ and $300 \mathrm{pc}$. Models are constructed for the same four values of $\tau_{20}$ and spectra are obtained for varying inclinations.

In Fig. 15 we show several fits to the spectrum of Cygnus A, with different variables. A small scaling factor is needed to fit the models, which is either due to a different intrinsic luminosity, or different value of $h_{0}$, which are both kept fixed.

\subsubsection{Tuning the fit: Size of the disk}

The size of the disk has the largest effect on the width of the SED. Good fits are obtained for all optical depths for a disk with $r=10-100 \mathrm{pc}$. The reference model does not fit the $\mathrm{SED}$, as expected from the colour-colour analysis. It overproduces near-infrared emission, and cannot account for all the far-infrared emission. The large and outer disk can fit the farinfrared output of Cygnus A, but both underpredict the observed mid-infrared flux. The inner radius of the disk can be carefully tuned to the shape and intensity of the mid-infrared observations. For an inner radius of $10 \mathrm{pc}$ the mid-infrared is well fitted by the model.

With an outer radius of $30 \mathrm{pc}$ all four mid-infrared points are well fitted by the different opacity models. The model with an outer radius of $100 \mathrm{pc}$ fits all the data, although the fit in the mid-infrared is somewhat worse. The longest wavelength farinfrared point is only fitted by the $10-100 \mathrm{pc}$ disk with $\tau_{20}=5$. Increasing the outer radius to $300 \mathrm{pc}$ improves the fit for other opacities at the longest wavelengths, but then the fit in the midinfrared deteriorates. The models with an outer radius of $100 \mathrm{pc}$ or more peak at $60 \mu \mathrm{m}$, as observed, and the slope of the farinfrared and sub-millimeter tail is reasonably consistent with the observations.

We have no problem reproducing the width of the observed SED with a single large torus model, as did previous models. However, from optical imaging and spectropolarimetry we know that Cygnus A has large scale dust and star-formation (Jackson et al. 1998; Fosbury et al. 1999), which is expected to contribute to the far-infrared emission. From the colour-colour analysis we also conclude that the far-infrared emission beyond $60 \mu \mathrm{m}$ is generally not produced by a torus. Therefore, the $10-30 \mathrm{pc}$ disk provides the best model for the torus in Cygnus A.

\subsubsection{Tuning inclination and opacity}

Small variations in inclination have little effect on the resulting SED and models with inclinations between 5 and 60 degrees fit equally well. Models with higher inclinations are ruled out.

The opacity cannot be fully constrained from the models. For the $10-100 \mathrm{pc}$ disk the $\tau_{20}=10$ and 20 models fit best, which is also the case in the $10-30 \mathrm{pc}$ disk. However, in the latter the opacity is not constrained by the far-infrared, which leaves only four datapoints to optimize the fit. The effect of 

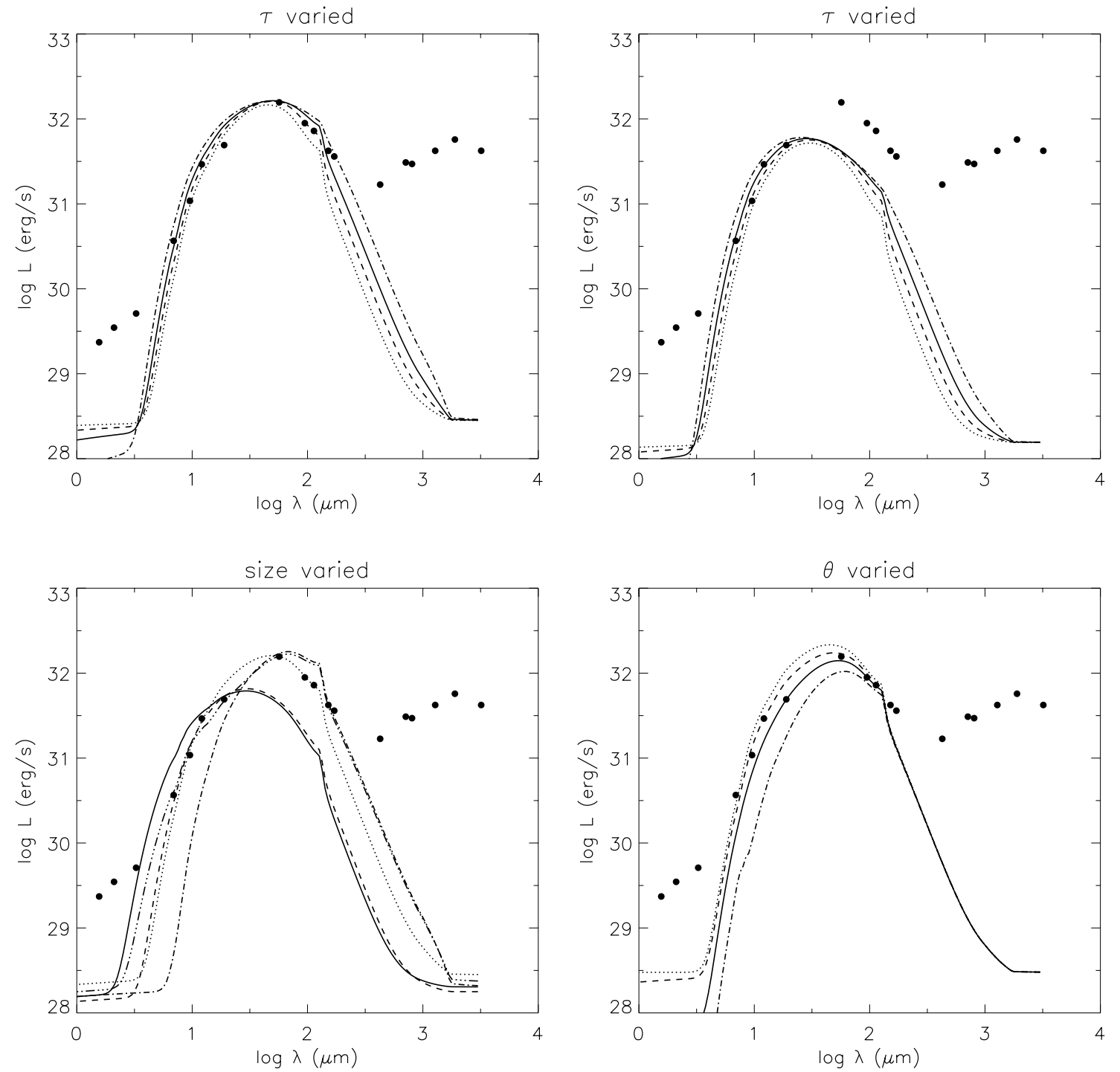

Fig. 15. Upper left panel: model fits of a conical disk to the observed SED of Cygnus A. The error bars on the observed luminosities are smaller than the plot symbols. The model disk extends from 10 to $100 \mathrm{pc}$ from the nucleus and has an inclination of $50^{\circ}$. Lines are for different opacity models $\left(\tau_{20}\right): 5=$ dotted, $10=$ dashed, $20=$ solid and $50=$ dash-dotted. Upper right panel: as left panel, but for a disk from 10-30 pc. Lower left panel: fits of disks with $\tau_{20}=10$ and inclination of 50 degrees. Lines are for different sizes: dotted $=10-100 \mathrm{pc}$, dashed $=10-30 \mathrm{pc}$, solid $=3-30 \mathrm{pc}$, dash-dot $=30-300 \mathrm{pc}$, dash-triple dot $=3-300 \mathrm{pc}$. Lower right panel: fits of disks with $\tau_{20}=10$, and size 10 to 100 pc. Different lines are for different inclinations. From top to bottom: 30 (dotted), 50 (dashed), 60 (solid) and 70 (dash-dotted). All panels have $\alpha=1$ and $\beta=0$ models.

varying $\alpha$ and $\beta$ has been described above, and is similar in the Cygnus A models. Changing them will not significantly improve the fit.

\subsubsection{Conclusions from the Cygnus $A$ fit}

From the observations of Cygnus A we can constrain the size and inclination of the obscuring torus. The geometry and density distribution are fixed, varying these parameters would not significantly change the output spectrum. Assuming significant star-formation in the host galaxy produces the far-infrared emission, the best fit for the torus is obtained for a disk with size of $10-30 \mathrm{pc}$, inclination of 50 degrees and optical depth of 10-20. A single large disk fits all infrared points, but does not allow a contribution from the observed large scale dust, and is therefore not fully consistent with optical observations of young stars and large scale dust in Cygnus A.

\section{Conclusions}

Radiative transfer models have been constructed to study the emission of a dusty torus around an active galactic nucleus. We have varied optical depth, disk geometry, disk size, density distribution and dust grain size distribution, to study the effect on the resulting SED. In most models, the dust becomes optically thin around $100 \mu \mathrm{m}$, which implies that the emission is isotropic at longer wavelengths. The models are compared to the observed infrared colours of a sample of radio-loud active galaxies, and furthermore fitted to observations of Cygnus A. The problem with previous models was often that the resulting 
SED was not broad enough. We have identified this as a matter of size and/or opacity: the larger the disk, or the higher the opacity, the broader the temperature distribution, and, as a consequence, the broader the spectrum. In the fit to Cygnus A we have no problem to reproduce the entire width of the observed infrared SED.

From the comparison to observed colours, we conclude that the tori in active galaxies are relatively large. The inner disk model does not fit any of the observations: the inner radius more likely lies around $10 \mathrm{pc}$. The outer radius is not well constrained. Pillbox models are rejected on basis of the colourcolour analysis, both the conical and flaring disks are possible. The surface density seems constant with radius; for more centrally concentrated models the colours are not consistent with observations. Varying the optical depth can sometimes improve the level of agreement, but its effect is diverse. An optimum value cannot be derived.

The objects that are best fitted by the models are the radio galaxies. In the hot vs. warm colours all broad-line radio galaxies fall entirely in the range of the model colours. This is consistent with observational evidence that in other objects a second dust component contributes, which is not related to a central obscuring torus. This second component alters the shape of the spectrum, and hence the colours, which explains the inconsistency of the models with the observed cold colours. We conclude that the torus dominates only at 12 and $25 \mu \mathrm{m}$. As a consequence, selecting active galaxies is best done using their 12 and/or $25 \mu \mathrm{m}$ power. However, at these wavelengths the quasars are expected to be brighter, for which bias one has to correct.

Hes et al. (1995) find a relation between the $60 \mu \mathrm{m}$ luminosity and radio power of active galaxies: the more powerful active galaxies are more luminous at $60 \mu \mathrm{m}$. They interpret this as evidence that the $60 \mu \mathrm{m}$ emission is dominated by reprocessed radiation from the obscuring torus. However, the above analysis suggests that the $60 \mu \mathrm{m}$ emission in active galaxies is dominated by a second component, and hence its power is not indicative of the power of the active nucleus. If star-formation dominates the $60 \mu \mathrm{m}$ emission in active galaxies, the correlation would imply that the more powerful active galaxies have higher star-formation rates. A better understanding of the process dominating the $60 \mu \mathrm{m}$ emission can be obtained by comparing individual fits to objects with and without star-formation, such as $3 \mathrm{C} 48$ and the broad-line radio galaxies.

For Cygnus A, the inner radius of the disk is constrained to lie around $10 \mathrm{pc}$. This value is found to be most consistent with the observations in the colour-colour plots. The outer radius can be anywhere within $30-300 \mathrm{pc}$, but the best fit is obtained with a value of $30-100 \mathrm{pc}$. All values are within the observational limits set by optical studies. Although a single torus model fits the entire infrared SED, there is strong observational evidence for a second dust component in Cygnus A. In addition, the quality of the fit in the mid-infrared improves somewhat for a smaller disk. An outer radius of $30 \mathrm{pc}$ is preferred to allow for the observed star-formation to contribute to the far-infrared emission in Cygnus A.

The consequences for unification models are ambiguous. On one hand, we can fit the entire SED of Cygnus A with a single torus model, while we know that other processes must contribute to its infrared emission. This is in itself evidence in favour of the presence of an obscuring torus. On the other hand, the cold colours of most objects are inconsistent with the torus models, indicating that a second component must be present, and a good fit to the entire SED is not possible. We know that this second component must also be present in Cygnus $\mathrm{A}$, and therefore, the relative contribution of star-formation must be assessed with other means to thoroughly disentangle the infrared SED of active galaxies. Only with a proper decomposition of the SED can the actual emission from an obscuring torus be studied in detail.

\section{Future work}

Infrared observations provide a powerful tool to study the emission from an obscuring torus in any type of active galaxy, especially at near- and mid-infrared wavelengths, where the emission from the torus is observed directly. The models presented in this paper are a next step in increasing our understanding of such tori. However, improvement of the models can be expected on several fronts.

As mentioned, the $10 \mu \mathrm{m}$ absorption feature is sensitive to the parameters of the model. Unfortunately, no data presently exist for radio-loud active galaxies, due to their faintness at mid-infrared data and lack of capable instruments. SIRTF is expected to have the sensitivity to observe a large sample of radio-loud active galaxies, over a large range of redshifts. By constructing models with higher frequency resolution around $10 \mu \mathrm{m}$, predictions can be made for future observations of $10 \mu \mathrm{m}$ emission or absorption in radio-loud active galaxies. In addition the spectral index of the mid-infrared spectrum is sensitive to the parameters, especially $\alpha_{12}^{25}$, and to some extent $\alpha_{25}^{60}$. Since this is the wavelength range where we are reasonably sure we observe emission from the torus, high resolution spectra are needed. The SIRTF satellite will be able to obtain spectra up to $90 \mu \mathrm{m}$ for active galaxies over a large range of redshifts, which will provide a good test for the models.

The models presented here are not optimal. Improved models could lead to a better understanding of the physics involved in the obscuring torus around an active nucleus. They could be made self-consistent with a scale height equal to the local radiative pressure scale height. The input spectrum could be adapted to better reflect the emission spectrum from an accretion disk, which often shows a strong bump in the soft X-rays. In addition, there is evidence that star-formation can occur in the torus, which will increase the scale height of the disk. We plan to take this into account as well. Finally, we have not treated indirect heating by the hot gas around the accretion disk. This gas absorbs the X-rays and re-emits the energy in the UV. This UV serves as an additional heating source for the dust. However, improvement of the models is only relevant when better observations are available to constrain the models, and when we have a better understanding of the contribution of star-formation to the infrared SED. 
Acknowledgements. The authors wish to thank Xander Tielens for initiating the collaboration and for advice and critical comments during the writing of the paper. Thanks to Peter Barthel for advice, comments and support, and thanks to Julian Krolik for critical discussions. Finally, we acknowledge expert reading by the referee, Željko Ivezić, leading to significant improvement of this paper and many new ideas for the future. CPD acknowledges support from the European Commission under TMR grant ERBFMRX-CT98-0195 ("Accretion onto black holes, compact objects and protostars").

\section{References}

Canalizo, G., \& Stockton, A. 2000, ApJ, 528, 201

Carilli, C. L., \& Barthel, P. D. 1996, A\&AR, 7, 1

Chiang, E. I., \& Goldreich, P. 1997, ApJ, 490, 368

Dullemond, C. P., \& Turolla, R. 2000, A\&A, 360, 1187

Efstathiou, A., \& Rowan-Robinson, M. 1995, MNRAS, 273, 649

Fosbury, R. A. E., Vernet, J., Villar-Martín, M., et al. 1999, in The Most Distant Radio Galaxies, 311

Golombek, D., Miley, G. K., \& Neugebauer, G. 1988, AJ, 95, 26

Granato, G. L., \& Danese, L. 1994, MNRAS, 268, 235
Heckman, T. M., Chambers, K. C., \& Postman, M. 1992, ApJ, 391, 39 Hes, R., Barthel, P. D., \& Hoekstra, H. 1995, A\&A, 303, 8 Imanishi, M., \& Ueno, S. 2000, ApJ, 535, 626

Jackson, N., Tadhunter, C., \& Sparks, W. B. 1998, MNRAS, 301, 131

Laor, A., \& Draine, B. T. 1993, ApJ, 402, 441

MacKenty, J. W. 1989, ApJ, 343, 125

Maiolino, R., Marconi, A., \& Oliva, E. 2001a, A\&A, 365, 37

Maiolino, R., Marconi, A., Salvati, M., et al. 2001b, A\&A, 365, 28

Mathis, J. S., Rumpl, W., \& Nordsieck, K. H. 1977, ApJ, 217, 425

Nenkova, M., Ivezić, Ž., \& Elitzur, M. 2002, ApJ, 570, L9

Neugebauer, G., Miley, G. K., Soifer, B. T., \& Clegg, P. E. 1986, ApJ, 308,815

Pier, E. A., \& Krolik, J. H. 1992, ApJ, 401, 99

Prieto, M. A., Pérez García, A. M., \& Rodríguez Espinosa, J. M. 2001, A\&A, 377, 60

Sekiguchi, K. 1987, ApJ, 316, 145

Spinoglio, L., Andreani, P., \& Malkan, M. A. 2002, ApJ, 572, 105

van Bemmel, I. M., \& Barthel, P. D. 2001, A\&A, 379, L21

van Bemmel, I. M., Barthel, P. D., \& de Graauw, T. 2000, A\&A, 359, 523

van Bemmel, I. M., Barthel, P. D., \& Yun, M. S. 1998, A\&A, 334, 799 